\title{
SW-Store: a vertically partitioned DBMS for Semantic Web data management
}

\author{
Daniel J. Abadi · Adam Marcus - Samuel R. Madden • \\ Kate Hollenbach
}

Received: 30 April 2008 / Revised: 1 November 2008 / Accepted: 3 November 2008 / Published online: 4 February 2009

(C) Springer-Verlag 2009

\begin{abstract}
Efficient management of RDF data is an important prerequisite for realizing the Semantic Web vision. Performance and scalability issues are becoming increasingly pressing as Semantic Web technology is applied to real-world applications. In this paper, we examine the reasons why current data management solutions for RDF data scale poorly, and explore the fundamental scalability limitations of these approaches. We review the state of the art for improving performance of RDF databases and consider a recent suggestion, "property tables". We then discuss practically and empirically why this solution has undesirable features. As an improvement, we propose an alternative solution: vertically partitioning the RDF data. We compare the performance of vertical partitioning with prior art on queries generated by a Web-based RDF browser over a large-scale (more than 50 million triples) catalog of library data. Our results show that a vertically partitioned schema achieves similar performance to the property table technique while being much simpler to design. Further, if a column-oriented DBMS (a database architected specially for the vertically partitioned case) is used instead of a row-oriented DBMS, another order of magnitude performance improvement is observed, with query times dropping from minutes to several seconds. Encouraged by these results, we describe the architecture of
\end{abstract}

\author{
D. J. Abadi $(\bowtie)$ \\ Yale University, New Haven, CT, USA \\ e-mail: dna@cs.yale.edu \\ A. Marcus $\cdot$ S. R. Madden $\cdot$ K. Hollenbach \\ MIT, Cambridge, MA, USA \\ e-mail: marcua@csail.mit.edu \\ S. R. Madden \\ e-mail: madden@csail.mit.edu \\ K. Hollenbach \\ e-mail:kjhollen@mit.edu
}

SW-Store, a new DBMS we are actively building that implements these techniques to achieve high performance RDF data management.

\section{Introduction}

The Semantic Web is an effort by the W3C [51] to enable integration and sharing of data across different applications and organizations. Though called the Semantic Web, the W3C envisions something closer to a global database than to the existing World-Wide Web. In the W3C vision, users of the Semantic Web should be able to issue structured queries over all of the data on the Internet, and receive correct and well-formed answers to those queries from a variety of different data sources that may have information relevant to the query. Database researchers will immediately recognize that building the Semantic Web requires surmounting many of the semantic heterogeneity problems faced by the database community over the years. In fact, as in many database research efforts, the $\mathrm{W} 3 \mathrm{C}$ has proposed schema matching, ontologies, and schema repositories for managing semantic heterogeneity.

One area in which the Semantic Web community differs from the relational database community is in its choice of data model. The Semantic Web data model, called the "Resource Description Framework" [38], or RDF, represents data as statements about resources using a graph connecting resource nodes and their property values with labeled arcs representing properties. Syntactically, this graph can be represented using XML syntax (RDF/XML). This is typically the format for RDF data exchange; however, structurally, the graph can be parsed into a series of triples, each representing a statement of the form < subject, property, object $>$, which is the notation we follow in this paper. These triples can then be 
stored in a relational database with a three-column schema. For example, to represent the fact that Serge Abiteboul, Rick Hull, and Victor Vianu wrote a book called "Foundations of Databases", we would use seven triples: ${ }^{1}$

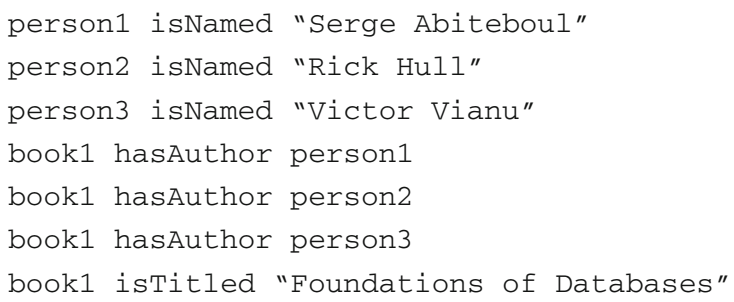

The commonly stated advantage of this approach is that it is very general (almost any type of data can be expressed in this format-it is easy to shred both relational and XML databases into RDF triples) and it is easy to build tools that manipulate RDF. These tools will not be useful if different users describe objects differently, so the Semantic Web community has developed a set of standards for expressing schemas (RDFS and OWL); these make it possible, for example, to say that every book should have an author, or that the property "isAuthor" is the same as the property "authored."

This data representation, though flexible, has the potential for serious performance issues, since there is only a single RDF table, and almost all interesting queries involve many self-joins over this table. For example, given the data table given at the top of Fig. 1, to find all of the authors of books whose title contains the word "Transaction" it is necessary to perform the five-way self-join query shown at the bottom of Fig. 1.

This query is potentially very slow to execute, since as the number of triples in the library collection scales, the RDF table may well exceed the size of memory, and each of these filters and joins will require a scan or index lookup. Real world queries involve many more joins, which complicates selectivity estimation and query optimization, and limits the benefit of indices.

As a database researcher, it is tempting to dismiss RDF, as the data model seems to offer inherently limited performance for little—or no-improvement in expressiveness or utility. Regardless of one's opinion of RDF, however, it appears to have a great deal of momentum in the web community, with several international conferences (ISWC, ESWC) each drawing more than 250 full paper submissions and several hundred attendees, as well as enthusiastic support from the W3C (and its founder, Tim Berners-Lee.) Furthermore, an increasing amount of data are becoming available on the Web in RDF format. For example, the UniProt comprehensive catalog of protein sequence, function, and annotation data

\footnotetext{
${ }^{1}$ In practice, RDF uses Universal Resource Identifiers (URIs), which look like URLs and often include sequences of numbers to make them unique. We use more readable names in our examples in this paper.
}

\begin{tabular}{|l|l|l|}
\hline subj & prop & obj \\
\hline ID1 & title & Transaction Processing \\
\hline ID2 & title & Database Management Systems \\
\hline ID1 & type & book \\
\hline ID2 & type & book \\
\hline ID3 & type & author \\
\hline ID4 & type & author \\
\hline ID5 & type & author \\
\hline ID6 & type & author \\
\hline ID3 & isnamed & Ramakrishnan \\
\hline ID4 & isnamed & Gehrke \\
\hline ID5 & isnamed & Gray \\
\hline ID6 & isnamed & Reuter \\
\hline ID1 & hasAuth & ID5 \\
\hline ID1 & hasAuth & ID6 \\
\hline ID2 & hasAuth & ID3 \\
\hline ID2 & hasAuth & ID4 \\
\hline
\end{tabular}

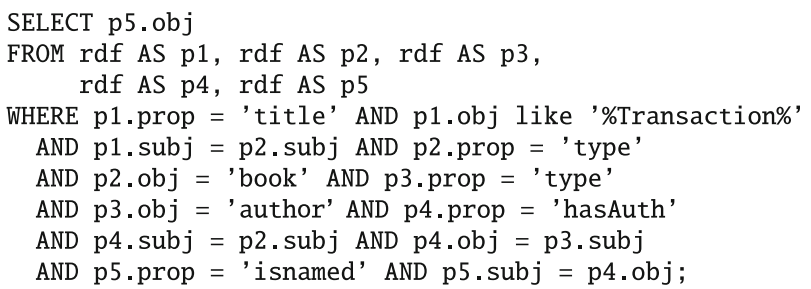

Fig. 1 Sample RDF data table (top) and SQL over a triple-store for a query that finds the names of all of the authors of books whose title contains the word "Transaction" (bottom)

(created by joining information contained in the Swiss-Prot, TrEMBL, and PIR databases) is now completely available in RDF [48] and contains over 600 million statements; the RDF version of the US census data (from year 2000) contain over 1 billion statements; and the W3C SWEO Linking Open Data community project [50] contains over 2 billion statements. Online Semantic Web search engine Sindice [44] claims to index over 100 million Semantic Web documents; similarly Falcons [22] claims to index 10 million Semantic Web documents, and Swoogle [46] 2.5 million Semantic Web documents.

Hence, it is our goal in this paper to explore ways to improve RDF query performance, since it appears that it will be an important way for people to represent data on (or about) the web. We focus on using a relational query processor to execute RDF queries, as we (and several other research groups $[18,19,24,32,54])$ feel that this is likely to be the best performing approach. The gist of our technique is based on a simple and familiar observation to proponents of relational technology: just as with relations, RDF does not have to be a proposal for physical storage - it is merely a logical data model. RDF databases are free to store RDF data as they see fit-including in ways that offer much better performance than actually storing collections of triples in memory or on disk.

We look at two different physical organization techniques for RDF data. The first, called the property table technique, denormalizes RDF tables by physically storing them in a 
wider, flattened representation more similar to traditional relational schemas. One way to do this flattening is to find sets of properties that tend to be defined together [19,26,53]; i.e., clusters of subjects tend to have these properties defined. For example, "title," "author," and "isbn" might all be properties that tend to be defined for subjects that represent book entities. Thus a table containing "subject" as the key and "title," "author," and "isbn" as the other attributes might be created to store entities of type "book." This flattened property table representation will require many fewer joins to access, since self-joins on the subject column can be eliminated. One can use standard query rewriting techniques to translate queries over the RDF triple-store to queries over the flattened representation.

There are several issues with this property table technique, including:

NULLs. Because not all properties will be defined for all subjects in the subject cluster, wide tables will have (possibly many) NULLs. For very wide tables with many sparse attributes, the space overhead of these NULLs can potentially dominate the space of the data itself.

Multi-valued Attributes. Multi-valued attributes (such as a book with multiple titles) and many-to-many relationships (such as the book authorship relationship where a book can have multiple authors and an author can write multiple books) are somewhat awkward to express in a flattened representation. Anecdotally, many RDF datasets make heavy use of multi-valued attributes, so this may be of more concern here than in other database applications.

Complexity. A clustering algorithm must typically be used to find groups of properties that tend to be defined together, or classes of subjects with similar property definitions. Furthermore, reclustering might become necessary as the data characteristics change over time.

To address these limitations, we propose a different physical organization technique for RDF data. We create a twocolumn table for each unique property in the RDF dataset where the first column contains subjects that define the property and the second column contains the object values for those subjects. For the library example, tables would be created for, e.g., the "title," "author," and "isbn," properties; each table listing subject URIs with their corresponding value for that property. Multi-valued subjects are thus represented as multiple rows in the table with the same subject and different values. Although many joins are required to answer queries over multiple properties, each table is sorted by subject, so fast (linear) merge joins can be used. Further, only those properties that are accessed by the query need to be read off disk (or from memory), saving I/O time.

The above technique can be thought of as a fully vertically partitioned database on property value. Although vertically partitioning a database can be done in a normal DBMS, these databases are not optimized for these narrow schemas (for example, the tuple header dominates the size of the actual data resulting in table scans taking 4-5 times as long as they need to), and there has been a large amount of recent work on column-oriented databases [3,15,16,33,45], which are DBMSs optimized for vertically partitioned schemas.

In this paper, we compare the performance of different RDF storage schemes on a real world RDF dataset. We use the Postgres open source DBMS to show that both the property table and the vertically partitioned approaches outperform the standard triple-store approach by more than a factor of 2 (average query times go from around $100 \mathrm{~s}$ to around $40 \mathrm{~s}$ ) and have superior scaling properties. We then show that one can get another order of magnitude in performance improvement by using a column-oriented DBMS since they are designed to perform well on vertically partitioned schemas (queries now run in an average of $3 \mathrm{~s}$ ). As a result of the good performance of the column-oriented DBMS, we describe the architecture of SW-Store, an extension of an open-source column-oriented DBMS, that is designed for high performance RDF data management and is currently being built.

The main contributions of this paper are the following: an overview of the state of the art for storing RDF data in databases, a proposal to vertically partition RDF data as a simple way to improve RDF query performance relative to the state of the art, a description of how we extended a column-oriented database to implement the vertical partitioning approach, and a performance evaluation of these different proposals. Ultimately, the column-oriented DBMS is able to obtain near-interactive performance (on non-trivial queries) over real-world RDF datasets of many millions of records, something that (to the best of our knowledge) no other RDF store has been able to achieve.

The remainder of this paper is organized as follows. In Sect. 2 we discuss the state of the art of storing RDF data in relational databases, with an extended look at the property table approach. In Sect. 3, we discuss the vertically partitioned approach and explain how this approach can be implemented inside a column-oriented DBMS. In Sect. 4 we look at an additional optimization to improve performance on RDF queries: materializing path expressions in advance. In Sect. 5, we summarize the library benchmark we use for evaluating the performance of an RDF database, and then compare the performance of the different RDF storage approaches in Sect. 6. These results lead into Sect. 7, where we present the architecture of SW-Store. Finally, we conclude in Sect. 8.

\section{Current state of the art}

In this section, we discuss the state of the art of storing RDF data in relational databases, with an extended look at the property table approach. 


\subsection{RDF in RDBMSs}

Although there have been non-relational DBMS proposals for storing RDF data [17], the majority of RDF data storage solutions use relational DBMSs, such as Jena [54], Oracle[19], Sesame [18], 3store [24], and SOR [31]. These solutions generally center around a giant triples table, containing one row for each statement. For example, the RDF triples table for a small library dataset is shown in Table 1a.

Since URIs and literal values tend to be long strings (rather than those shown in the simplified example in Table 1a), many RDF stores choose not to store entire strings in the triples table; instead they store shortened versions or keys. Oracle and Sesame map string URIs to integer identifiers so the data is normalized into two tables, one triples table using identifiers for each value, and one mapping table that maps the identifiers to their corresponding strings. This can be thought of as dictionary encoding the string data. 3store does something similar, except the identifiers are created by applying a hash function to each string. Jena prefers to just dictionary encode the namespace prefix part of the URI and only normalizes particularly long URIs into a separate table.

Each of the above listed RDF storage solutions implements a multi-layered architecture, where RDF-specific functionality (for example, query translation) is performed in a layer above the RDBMS (which sits in the lowest layer). This removes any dependence on the particular RDBMS used (though Sesame will take advantage of specific features of an object relational DBMS such as Postgres to use subtables to model class and property subsumption relations). Queries are issued in an RDF-specific querying language (such as SPARQL [41] or RDQL [39]), converted to SQL in the higher level RDF layers, and then sent to the RDBMS which will optimize and execute the SQL query over the triple-store.

For example, the SPARQL query that attempts to get the title of the book(s) Joe Fox wrote in 2001:

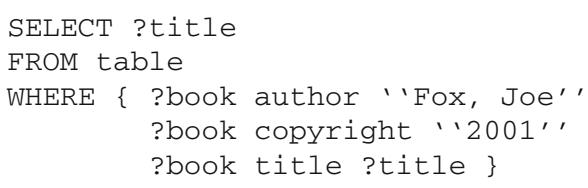

would get converted into the SQL query shown in Table $1 \mathrm{~b}$ run over the data in Table 1a.

Note that this simple query results in a three-way self-join over the triples table (in fact, another join will generally be needed if the strings are normalized into a separate table, as described above). If the predicates are selective, this 3-way join is not expensive (assuming the filters can be pushed down into the query [30] and that the triples table is indexedtypically there will be indexes on all three columns). However, the less selective the predicates, the more problematic the joins become. As a result, both Jena and Oracle propose

changes to the schema to reduce the number of joins of this type: property tables. We now examine these data structures in more detail.

\subsection{Property tables}

Researchers developing the Jena Semantic Web toolkit, Jena2 $[53,54]$ proposed the use of property tables to speed up queries over triple-stores. They proposed two types of property tables. The first type, which we call a clustered property table, contains clusters of properties that tend to be defined together. For example, for the raw data in Table 1a, type, title, and copyright date tend to be defined as properties for similar subjects. Thus, a property table containing these three properties as attributes along with subject as the table key can be created, which stores the triples from the original data whose property is one of these three attributes. The resulting property table, along with the left-over triples that are not stored in this property table, is shown in Table 1c. Multiple property tables with different clusters of properties may be created; however, a key requirement for this type of property table is that a particular property may only appear in at most one property table.

The second type of property table, termed a propertyclass table, exploits the type property of subjects to cluster

Table 1 Some sample RDF data and possible property tables

\begin{tabular}{|c|c|c|}
\hline Subj. & Prop. & Obj. \\
\hline ID1 & type & BookType \\
\hline ID1 & title & "XYZ" \\
\hline ID1 & author & "Fox, Joe" \\
\hline ID1 & copyright & "2001" \\
\hline ID2 & type & CDType \\
\hline ID2 & title & "ABC" \\
\hline ID2 & artist & "Orr, Tim" \\
\hline ID2 & copyright & "1985" \\
\hline ID2 & language & "French" \\
\hline ID3 & type & BookType \\
\hline ID3 & title & "MNO" \\
\hline ID3 & language & "English" \\
\hline ID4 & type & DVDType \\
\hline ID4 & title & "DEF" \\
\hline ID5 & type & CDType \\
\hline ID5 & title & "GHI" \\
\hline ID5 & copyright & "1995" \\
\hline ID6 & type & BookType \\
\hline ID6 & copyright & "2004" \\
\hline
\end{tabular}

(a) Some Example RDF Triples

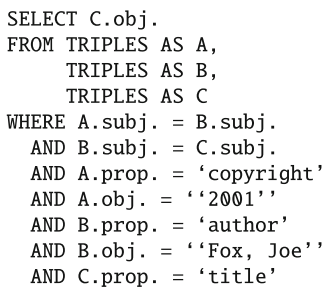

(b) Example SQL Query Over RDF Triples Table From (a)

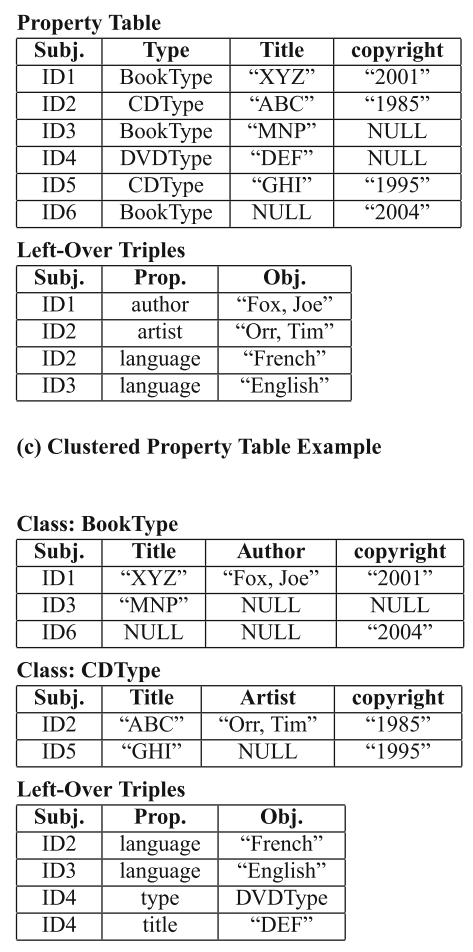

(d) Property-Class Table Example 
similar sets of subjects together in the same table. Unlike the first type of property table, a property may exist in multiple property-class tables. Table $1 \mathrm{~d}$ shows two example property tables that may be created from the same set of input data as Table $1 \mathrm{c}$. Jena 2 found property-class tables to be particularly useful for the storage of reified statements (statements about statements) where the class is rdf:Statement and the properties are rdf:Subject, rdf:Property, and rdf:Object.

Oracle [19] also adopts a property table-like data structure (they call it a "subject-property matrix") to speed up queries over RDF triples. Their utilization of property tables is slightly different from Jena2 in that they are not used as a primary storage structure, but rather as an auxiliary data structure - a materialized view - that can be used to speed up specific types of queries.

The most important advantage of the introduction of property tables to the triple-store is that they can reduce subjectsubject self-joins of the triples table. For example, the simple query shown in Sect. 2.1 ("return the title of the book(s) Joe Fox wrote in 2001") resulted in a three-way self-join. However, if title, author, and copyright were all located inside the same property table, the query can be executed via a simple selection operator.

Another advantage of property tables is that they open up the possibility for attribute typing. Rather than storing objects of many different types in a single column in a triple-store (and thus in string literal format), attributes like copyright can be stored in specific date format. This saves space and can speed up certain operations such as numerical functions, aggregations, or comparison operations within index code.

To the best of our knowledge, property tables have not been widely adopted except in specialized cases (like reified statements). One reason for this may be that they have a number of disadvantages. As Wilkinson points out [53], one disadvantage of property tables is that while they are very good at speeding up queries that can be answered from a single property table, most queries require joins or unions to combine data from several tables. For example, for the data in Table 1, if a user wishes to find out if there are any items in the catalog copyrighted before 1990 in a language other than English, the following SQL queries could be issued:

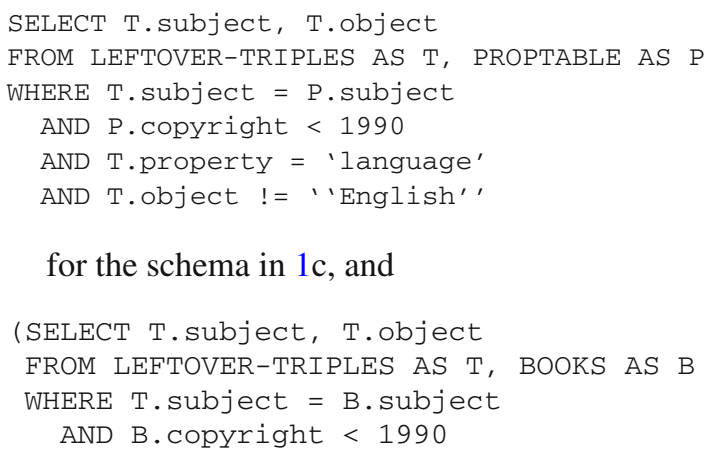

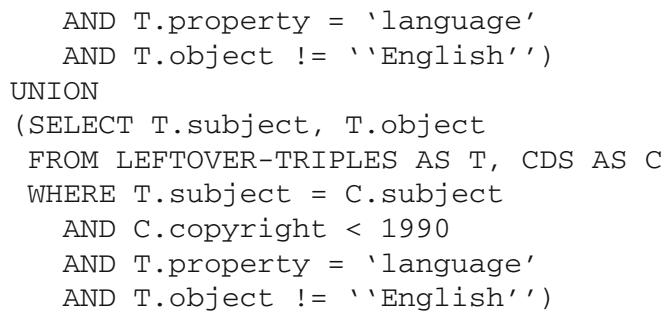

for the schema in 1d. As can be seen, join and union clauses get introduced into the queries, and query translation and plan generation get complicated very quickly. Queries that do not select on class type are generally problematic for property-class tables, and queries that have unspecified property values (or for whom property value is bound at run-time) are generally problematic for clustered property tables.

Another disadvantage of property tables is that RDF data tends not to be very structured, and not every subject listed in the table will have all the properties defined. The less structured the data, the more NULL values will exist in the table. In fact, these representations can be extremely sparsecontaining hundreds of NULLs for each non-NULL value. These NULLs impose a substantial performance overhead, as has been noted in previous work $[2,7,12]$.

The two problems with property tables are at odds with one another. If property tables are made narrow, with few property columns that are highly correlated in their value definition, the average value density of the table increases and the table is less sparse. Unfortunately, the likelihood of any particular query being able to be confined to a single property table is reduced. On the other hand, if many properties are included in a single property table, the number of joins and union clauses per query decreases, but the number of NULLs in the table increases (it becomes more sparse), bloating the table and wasting space. Thus there is a fundamental trade-off between query complexity as a result of proliferation of joins and unions and table sparsity (and its resulting impact on query performance). Similar problems have been noted in attempts to shred and store XML data in relational databases $[23,43]$.

A third problem with property tables is the abundance of multi-valued attributes found in RDF data. Multi-valued attributes are surprisingly prevalent in the Semantic Web; for example in the library catalog data we work with in Sect. 5, properties one might think of as single-valued such as title, publisher, and even entity type are multi-valued. In general, there always seem to be exceptions, and the RDF data model provides no disincentives for making properties multivalued. Furthermore, our experience suggests that RDF data often has overloaded subject URIs, where the same URI is used to represent many different real-world entities.

Multi-valued properties are problematic for property tables for the same reason they are problematic for relational 
tables. They cannot be included with the other attributes in the same table unless they are represented using list, set, or bag attributes. However, this requires an object-relational DBMS, results in variable width attributes, and complicates the expression of queries over these attributes.

In summary, while property tables can significantly improve performance by reducing the number of self-joins and typing attributes, they introduce complexity by requiring property clustering to be carefully done to create property tables that are not too wide, while still being wide enough to answer most queries directly. Ubiquitous multi-valued attributes cause further complexity.

\section{A simpler alternative}

We now look at an alternative to the property table solution to speed up queries over a triple-store. In Sect. 3.1, we discuss the vertically partitioned approach to storing RDF triples. We then look at how we extended a column-oriented DBMS to implement this approach in Sect. 3.2.

\subsection{Vertically partitioned approach}

We propose storage of RDF data using a fully decomposed storage model (DSM) [20], a performance enhancing technique that has proven to be useful in a variety of applications including data warehousing [2], biomedical data [21], and in the context of RDF/S, for taxonomic data $[9,47]$. The triples table is rewritten into $n$ two-column tables where $n$ is the number of unique properties in the data. In each of these tables, the first column contains the subjects that define that property and the second column contains the object values for those subjects. For example, the triples table from Table 1a would be stored as:

\begin{tabular}{|c|c|}
\hline \multicolumn{2}{|c|}{ Type } \\
\hline ID1 & BookType \\
\hline ID2 & CDType \\
\hline ID3 & BookType \\
\hline ID4 & DVDType \\
\hline ID5 & CDType \\
\hline ID6 & BookType \\
\hline \multicolumn{2}{|c|}{ Author } \\
\hline ID1 & "Fox, Joe" \\
\hline
\end{tabular}

\begin{tabular}{|l|c|}
\multicolumn{1}{|c|}{ Title } \\
\hline ID1 & "XYZ" \\
\hline ID2 & "ABC" \\
\hline ID3 & "MNO" \\
\hline ID4 & "DEF" \\
\hline ID5 & "GHI" \\
\hline \multicolumn{2}{|c|}{ Artist } \\
\hline ID2 & "Orr, Tim" \\
\hline
\end{tabular}

Copyright
\begin{tabular}{|c|c|}
\hline ID1 & "2001" \\
\hline ID2 & "1985" \\
\hline ID5 & "1995" \\
\hline ID6 & "2004" \\
\hline \multicolumn{2}{|c|}{ Language } \\
\hline ID2 & "French" \\
\hline ID3 & "English" \\
\hline
\end{tabular}

Each table is sorted by subject, so that particular subjects can be located quickly, and that fast merge joins can be used to reconstruct information about multiple properties for subsets of subjects. The value column for each table can also be optionally indexed (or a second copy of the table can be created, that is clustered on the value column).

The advantages of this approach (relative to the property table approach) are:

Support for multi-valued attributes. A multi-valued attribute is not problematic in the decomposed storage model.
If a subject has more than one object value for a particular property, then each distinct value is listed in a successive row in the table for that property. For example, if ID1 had two authors in the example above, the table would look like:

\begin{tabular}{|l|c|}
\multicolumn{2}{c}{ Author } \\
\hline ID1 & "Fox, Joe" \\
\hline ID1 & "Green, John" \\
\hline
\end{tabular}

Support for heterogeneous records. Subjects that do not define a particular property are simply omitted from the table for that property. In the example above, author is only defined for one subject (ID1) so the table can be kept small (NULL data need not be explicitly stored). The advantage becomes increasingly important when the data is not well structured.

Only those properties accessed by a query need to be read. I/O costs can be substantially reduced.

No clustering algorithms are needed. This point is the basis behind our claim that the vertically partitioned approach is simpler than the property table approach. While property tables need to be carefully constructed so that they are not too wide, but yet wide enough to independently answer queries, the algorithm for creating tables in the vertically partitioned approach is straightforward and need not change over time.

Fewer unions (relative to the property-class schema approach). In the property-class schema approach (see Fig. 1d), queries that do not restrict on class tend to have many union clauses. Since, in the vertically partitioned approach, all data for a particular property is located in the same table, union clauses in queries are less common.

Of course, there are several disadvantages to this approach.

Increased number of joins. When a query accesses several properties, multiple two-column vertical partitions have to be merged. Although this merge process can be performed by using simple, fast (linear) merge joins and is therefore not very expensive, the process is also not free. One particularly problematic class of queries are queries that do not restrict on property value (or, similarly, the value of the property will be bound only when the query is processed). In this situation, all vertical partitions need to be accessed and unioned together or merged: this class of queries is also problematic for property tables; however, since there are fewer total property tables than vertical partitions, the relative performance overhead of unioning or merging everything together is more significant for the vertical partitioning approach.

Although queries that do not restrict on property value are common in synthetic SPARQL benchmarks [42], we have found that, in practice, for the RDF applications we have worked with, such queries are rare. However, should an application contain queries that do not restrict on property value, the vertical partitions can be supplemented with 
auxiliary data structures that store data in a way that can accelerate this class of queries. One example of such auxiliary data structures is presented in the recent Hexastore paper [52], which, in addition to storing one vertical partition per property, also stores vertical partitions for subjects and objects as well. Hence, queries that do not restrict on property, but have selective predicates on subject or object can be executed quickly.

Inserts. Inserts can be slow for vertically partitioned schemas, since inserted statements about the same subject end up needing to access multiple different partitions (one per property that is defined for the inserted subject). Each partition access can result in a disk seek in the worst case. However, for all RDF applications we have built, we have found that the insert rate is low enough that buffering the inserts in memory and batch rewriting the tables performs well (see Sect. 7.5 for a description of this batching technique). We believe this will be the case for many RDF applications.

In Sect. 6 we will compare the performance of the property table approach and the vertically partitioned approach to each other and to the triples table approach. Before we present these experiments, we describe how a column-oriented DBMS can be extended to implement the vertically partitioned approach.

\subsection{Extending a column-oriented DBMS}

The fundamental idea behind column-oriented databases is to store tables as collections of columns rather than as collections of rows. In standard row-oriented databases (e.g., Oracle, DB2, SQLServer, Postgres, etc.) entire tuples are stored consecutively (either on disk or in memory). The problem with this is that if only a few attributes are accessed per query, entire rows need to be read into memory from disk (or into cache from memory) before the projection can occur, wasting bandwidth. By storing data in columns rather than rows (or in $n$ two-column tables for each attribute in the original table as in the vertically partitioned approach described above), projection occurs for free-only those columns relevant to a query need to be read. On the other hand, inserts might be slower in column-stores, especially if they are not done in batch.

Although the column-store idea has been around for some time [11], there has been a recent revival in column-oriented research and commercial products $[15,16,33,45]$. This can be attributed to the increasing divergence of CPU speeds relative to memory and disk access speeds [14]. As the speed gap widens, the wasted bandwidth utilization observed in roworiented DBMSs becomes increasingly costly. Additionally, as read-mostly/batch-write database applications (such as data warehousing) become more prevalent, so will columnoriented databases. Data warehousing database software

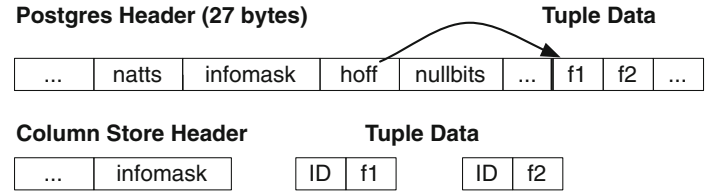

Fig. 2 Postgres headers (top) versus column-store headers (bottom). Column-stores store their headers in a separate column

contains $\$ 3.98$ billion [49] of the $\$ 14.6$ billion database market [36] (27\%) and is growing at a rate of $10.3 \%$ annually [49].

At first blush, it might seem strange to use a columnstore to store a set of two-column tables since column-stores excel at storing big wide tables where only a few attributes are queried at once. However, column-stores are actually wellsuited for schemas of this type, for the following reasons:

- Tuple headers are stored separately. Databases generally store tuple metadata at the beginning of the tuple. For example, Postgres contains a 27 byte tuple header containing information such as insert transaction timestamp, number of attributes in tuple, and NULL flags. In contrast, the rest of the data in the two-column tables will generally not take up more than 8 bytes (especially when strings have been dictionary encoded). A columnstore puts header information in separate columns and can selectively ignore it (a lot of this data is no longer relevant in the two column case; for example, the number of attributes is always two, and there are never any NULL values since subjects that do not define a particular property are omitted). Thus, the effective tuple width in a column-store is on the order of 8 bytes, compared with 35 bytes for a row-store like Postgres, which means that table scans perform four to five-times quicker in the column-store. Figure 2 illustrates the headers from Postgres and a typical column-store.

- Optimizations for fixed-length tuples. In a row-store, if any attribute is variable length, then the entire tuple is variable length. Since this is the common case, row-stores are designed for this case, where tuples are located through pointers in the page header (instead of address offset calculation) and are iterated through using an extra function call to a tuple interface (instead of iterated through directly as an array). This has a significant performance overhead $[15,16]$. In a column-store, fixed length attributes are stored as arrays. For the two-column tables in our RDF storage scheme, both attributes are fixed-length (assuming strings are dictionary encoded).

- Column-oriented data compression. In a column-store, since each attribute is stored separately, each attribute can be compressed separately using an algorithm best 
suited for that column. This can lead to significant performance improvement [4]. For example, the subject ID column, a monotonically increasing array of integers, is very compressible. Data from the same attribute come from the same attribute domain, and thus tend to have a higher degree of data value locality. Further, domain specific compression techniques can be used to compress the attribute data and it is often possible to operate directly on this representation. Bandwidth requirements are reduced when transferring compressed data, further improving performance.

- Carefully optimized column merge code. Since merging columns is a very frequent operation in column-stores, the merging code is carefully optimized to achieve high performance [6]. For example, extensive prefetching is used when merging multiple columns, so that disk seeks between columns (as they are read in parallel) do not dominate query time. Merging tables sorted on the same attribute can use the same code as merging columns from the same table.

- Direct access to sorted files rather than indirection through a B tree. While not strictly a property of column-oriented stores, the increased dependence on merge joins necessitates that heap files are maintained in guaranteed sorted order, whereas the order of heap files in many row-stores, even on a clustered attribute, is only guaranteed through an index. Thus, iterating through a sorted file must be done indirectly through the index, and extra seeks between index leaves may degrade performance.

- Column-oriented query optimizer. The decision as to whether to push selections past merge joins when an attribute is reaccessed later on in a query plan is not obvious and can affect query performance by an order of magnitude [6]. Having an optimizer designed for attributeoriented storage can further improve performance.

In summary, a column-store vertically partitions attributes of a table. The vertically partitioned scheme described in Sect. 3.1 can be thought of as partitioning attributes from a wide universal table containing all possible attributes from the data domain. Consequently, it makes sense to use a DBMS that is optimized for this type of partitioning.

\subsubsection{Implementation details}

We extended an open source column-oriented database system (C-Store [45]) to experiment with the ideas presented in this paper. C-Store stores a table as a collection of columns, each column stored in a separate file. Each file contains a list of $64 \mathrm{~K}$ blocks with as many values as possible packed into each block. C-Store, as a bare-bones research prototype, did not have support for temporary tables, index-nested loops join, union, or operators on the string data type at the outset of this project, each of which had to be added. We chose to dictionary encode strings similarly to Oracle and Sesame (as described in Sect. 2.1) where only fixed-width integer keys are stored in the data tables, and the keys are decoded at the end of each query using an index-nested loops join with a large strings dictionary table.

\section{Materialized path expressions}

In all three RDF storage schemes described thus far (triples schema, property tables, and vertically partitioned tables), querying path expressions (a common operation on RDF data) is expensive. In RDF data, object values can either be literals (e.g., "Fox, Joe") or URIs (e.g., http://preamble/FoxJoe). In the latter case, the value can be further described using additional triples (e.g., <BookID1, Author, http://preamble/FoxJoe >, < http://preamble/ FoxJoe, wasBorn, "1860" $>$ ). If one wanted to find all books whose authors were born in 1860 , this would require a path expression through the data. In a triples store, this query might look like

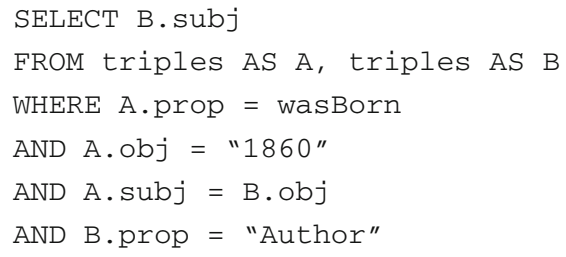

We need to perform a subject-object join to connect information about authors with information on the books they wrote.

In general, in a triples schema, a path expression requires $(n-1)$ subject-object self-joins where $n$ is the length of the path. For a property table schema, $(n-1)$ self-joins are also required if all properties in the path expression are included in the table; otherwise the property table needs to be joined with other tables. For the vertically partitioned schema, the tables for the properties involved in the path expression need to be joined together; however these are joins of the second (unsorted) column of one table with the first column of the other table (and are hence not merge joins).

Graphically, the data are modeled as shown in Fig. 3a. Here we use the standard RDF semantic model where subjects and objects are connected by labeled directed edges (properties). The path expression join can be observed through the author and wasBorn properties. If we could store the results of following the path expression through a more direct path (shown in Fig. 3b), the join could be eliminated: 


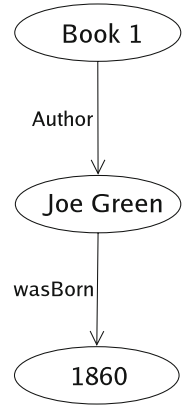

(a)

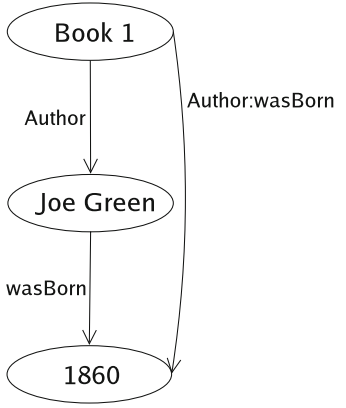

(b)
Fig. 3 Graphical presentation of subject-object join queries

SELECT A.subj

FROM proptable AS A,

WHERE A. author: wasBorn = "1860"

Using a vertically partitioned schema, this author:wasBorn path expression can be precalculated (similarly to how path indexes work in OODBMSs $[13,27,35]$ ) and the result stored in its own two column table as if it were a regular property. By precalculating the path expression, we do not have to perform the join at query time. Note that if any of the properties along the path in the path expression were multi-valued, the result would also be multi-valued. Thus, this materialized path expression technique is easier to implement in a vertically partitioned schema than in a property table.

Inference queries (e.g., if $\mathrm{X}$ is a part of $\mathrm{Y}$ and $\mathrm{Y}$ is a part of $Z$ then $X$ is a part of $Z$ ), a very common operation on Semantic Web data, are also usually performed using subject-object joins, and can be accelerated through this method.

There is, however, a cost in having a larger number of extra materialized tables, since they need to be recalculated whenever new triples are added to the RDF store. Thus, for read-only or read-mostly RDF applications, many of these materialized path expression tables can be created, but for insert heavy workloads, only very common path expressions should be materialized.

Although the materialization step is not an automatic improvement that comes with the presented architectures, both the property table and the vertically partitioning approaches naturally extend to allowing such calculations to be precomputed if they appear on a common path expression.

\section{Benchmark}

In this section, we describe the RDF benchmark we have developed for evaluating the performance of our three RDF databases. Our benchmark is based on publicly available library data and a collection of queries generated from a webbased user interface for browsing RDF content.

\subsection{Barton data}

The dataset we work with is taken from the publicly available Barton Libraries dataset [28]. These data are provided by the Simile Project [40], which develops tools for library data management and interoperability. The data contain records acquired from an RDF-formatted dump of the MIT Libraries Barton catalog, converted from raw data stored in an old library format standard called Machine Readable Catalog (MARC). Because of the multiple sources the data were derived from and the diverse nature of the data that is cataloged, the structure of the data is quite irregular.

We converted the Barton data from RDF/XML syntax to triples using the Redland parser [37] and then eliminated duplicate triples. We then did some very minor cleaning of data, eliminating triples with particularly long literal values or with subject URIs that were obviously overloaded to correspond to several real-world entities (more than $99 \%$ of the data remained). This left a total of 50, 255, 599 triples in our dataset, with a total of 221 unique properties, of which the vast majority appear infrequently. Of these properties, 82 (37\%) are multi-valued, meaning that they appear more than once for a given subject; however, these properties appear more often ( $77 \%$ of the triples have a multi-valued property). The dataset provides a good demonstration of the relatively unstructured nature of Semantic Web data.

\subsection{Longwell overview}

Longwell [29] is a tool developed by the Simile Project, which provides a graphical user interface for generic RDF data exploration in a web browser. The style of browsing employed, termed "faceted" browsing, where a user can quickly find data of interest by restricting various dimensions the data can take, is becoming an increasingly common avenue for interaction with RDF data [31]. The Longwell technology was recently deployed in Metaweb's Freebase Parallax tool [34].

A Longwell session begins by presenting the user with a list of the values the type property can take (such as Text or Notated Music in the library dataset) and the number of times each type occurs in the data. The user can then click on the types of data to further explore. Longwell shows the list of currently filtered resources (RDF subjects) in the main portion of the screen, and a list of filters in panels along the side. Each panel represents a property that is defined on the current filter, and contains popular object values for that property along with their corresponding frequencies. If the user selects an object value inside one of these property panels, this filters the working set of resources to those that have that property-object pair defined, updating the other panels with the new frequency counts for this narrower set of resources. 
We will now describe a sample browsing session through the Longwell interface. The reader may wish to follow the described path by looking at a set of screenshots taken from the online Longwell Demo we include in our companion technical report [1]. The path starts when the user selects Text from the type property box, which filters the data into a list of text entities. On the right side of the screen, we find that popular properties on these entities include "subject," "creator," "language," and "publisher." Within each property there is a list of the counts of the popular objects within this property. For example, we find out that the German object value appears 122 times and the French object value appears 131 times under the language property. By clicking on "fre" (French language), information about the 131 French texts in the database is presented, along with the revised set of popular properties and property values defined on these French texts.

Currently, Longwell only runs on a small fraction of the Barton data (9,375 records), as its RDF triple-store cannot scale to support the full 50 million triple dataset (we show this scalability limitation in our experiments). Our experiments use Longwell-style queries to provide a realistic benchmark for testing the designs proposed. Our goal is to explore architectures and schemas which can provide interactive performance on the full dataset.

By displaying only a subset of the properties in the display window at once, Longwell queries do not suffer from the unrestricted property problem mentioned in Sect. 3.1. One might argue that this is a drawback of the benchmark, as it does not contain an example of a class of queries this is particularly problematic for vertical partitioning. However, since our goal is to evaluate performance on a realistic benchmark, and since the unrestricted property problem can be solved using other methods [52], we choose not to synthetically introduce queries of this type.

\subsection{Longwell queries}

Our experiments feature seven queries that need to be executed on a typical Longwell path through the data. These queries are based on a typical browsing session, where the user selects a few specific entities to focus on and where the aggregate results summarizing the contents of the RDF store are updated.

The full queries are described at a high level here and are provided in full in the appendix as SQL queries against a triple-store. We will discuss later how we rewrote the queries for each schema.

Query 1 (Q1). Calculate the opening panel displaying the counts of the different types of data in the RDF store. This requires a search for the objects and counts of those objects with property Type.
There are 30 such objects. For example Type: Text has a count of 1,542,280, and Type: NotatedMusic has a count of 36,441.

Query 2 (Q2). The user selects Type: Text from the previous panel. Longwell must then display a list of other defined properties for resources of Type: Text. It must also calculate the frequency of these properties. For example, the Language property is defined $1,028,826$ times for resources that are of Type: Text.

Query 3 (Q3). For each property defined on items of Type: Text, populate the property panel with the counts of popular object values for that property (where popular means that an object value appears more than once). For example, the property Edition has eight items with value "[1st_ed._reprinted]."

Query 4 (Q4). This query recalculates all of the propertyobject counts from Q3 if the user clicks on the "French" value in the "Language" property panel. Essentially this is narrowing the working set of subjects to those whose Type is Text and Language is French. This query is thus similar to Q3, but has a much higher-selectivity.

Query 5 (Q5). Here we perform a type of inference. If there are triples of the form ( $X$ Records $Y$ ) and ( $Y$ Type $Z$ ) then we can infer that $X$ is of type $Z$. Here $X$ Records $Y$ means that $X$ records information about $Y$ (for example, $X$ might be a web page with information on $Y$ ). For this query, we want to find the inferred type of all subjects that have this Records property defined that also originated in the US Library of Congress (i.e., contain triples of the form ( $X$ origin “ $D L C$ ”)). The subject and inferred type is returned for all non-Text entities.

Query 6 (Q6). For this query, we combine the inference first step of Q5 with the property frequency calculation of Q2 to extract information in aggregate about items that are either directly known to be of Type: Text (as in Q2) or inferred to be of Type: Text through the Q5 Records inference.

Query 7 (Q7). Finally, we include a simple triple selection query with no aggregation or inference. The user tries to learn what a particular property (in this case Point) actually means by selecting other properties that are defined along with a particular value of this property. The user wishes to retrieve subject, Encoding, and Type of all resources with a Point value of "end." The result set indicates that all such resources are of the type Date. This explains why these resources can have "start" and "end" values: each of these resources represents a start or end date, depending on the value of Point.

We make the assumption that the Longwell administrator has selected a set of 28 interesting properties over which queries will be run (see discussion in the final paragraph of Sect. 5.2). These properties are listed in our technical 
report [1]. There are 26,761,389 triples for these properties. For queries Q2, Q3, Q4, and Q6, only these 28 properties are considered for aggregation.

\section{Evaluation}

Now that we have described our benchmark dataset and the queries that we run over it, we compare their performance in three different schemas-a triples schema, a property tables schema, and a vertically partitioned schema. We study the performance of each of these three schemas in a row-store (Postgres) and, for the vertically partitioned schema, also in a column-store (our extension of C-Store).

Our goal is to study the performance tradeoffs between these representations to understand when a vertically partitioned approach performs better (or worse) than the property tables solution. Ultimately, the goal is to improve performance as much as possible over the triple-store schema, since this is the schema most RDF store systems use.

\subsection{System}

Our benchmarking system is a hyperthreaded $3.0 \mathrm{GHz}$ Pentium IV, running RedHat Linux, with 2 Gbytes of memory, 1MB L2 cache, and a 3-disk, 750 Gbyte striped RAID array. The disk can read cold data at 150-180 MB/sec.

\subsubsection{PostgreSQL database}

We chose Postgres as the row-store to experiment with because Beckmann et al. [12] experimentally showed that it was by far more efficient dealing with sparse data than commercial database products. Postgres does not waste space storing NULL data: every tuple is preceded by a bit-string of cardinality equal to the number of attributes, with ' 1 's at positions of the non-NULL values in the tuple. NULL data is thus not stored; this is unlike commercial products that waste space on NULL data. Beckmann et al. show that Postgres queries over sparse data operate about eight times faster than commercial systems.

We ran Postgres with work $\_m e m=51200$, meaning that 50 Mbytes of memory are dedicated to each sorting and hashing operation. This may seem low, but the work_mem value is considered per operation, many of which are highly parallelizable. For example, when multiple aggregations are simultaneously being processed during the UNIONed GROUP BY queries for the property table implementation, a higher value of work_mem would cause the query executor to use all available physical memory and thrash. We set effective_cache_size to $1835004 \mathrm{~KB}$ pages. This value is a planner hint to predict how much memory is available in both the Postgres and operating system cache for overall caching. Setting it to a higher value does not change the plans for any of the queries run. We turned fsync off to avoid syncing the write-ahead log to disk to make comparisons to C-Store fair, since it does not use logging [45]. All queries were run at a READ COMMITTED isolation level, which is the lowest level of isolation available in Postgres, again because C-Store was not using transactions.

\subsection{Store implementation details}

We now describe the details of our store implementations. Note that all implementations feature a dictionary encoding table that maps strings to integer identifiers (as was described in Sect. 2.1); these integers are used instead of strings to represent properties, subjects, and objects. The encoding table has a clustered $\mathrm{B}+$ tree index on the identifiers, and an unclustered $\mathrm{B}+$ tree index on the strings. We found that all experiments, including those on the triple-store, went an order of magnitude faster with dictionary encoding.

\subsubsection{Triple store}

Of the popular full triple-store implementations, Sesame [18] seemed the most promising in terms of performance because it provides a native store that utilizes $\mathrm{B}+$ tree indices on any combination of subjects, properties, and objects, but does not have the overhead of a full database (of course, scalability is still an issue as it must perform many self-joins like all triple-stores). We were unable to test all queries on Sesame, as the current version of its query language, SeRQL, does not support aggregates (which are slated to be included in version 2 of the Sesame project). Because of this limitation, we were only able to test Q5 and Q7 on Sesame, as they did not feature aggregation. The Sesame system implements dictionary encoding to remove strings from the triples table, and including the dictionary encoding table, the triples table, and the indices on the tables, the system took 6.4 GBytes on disk.

On Q5, Sesame took 1400.94 s. For Q7, Sesame completed in $79.98 \mathrm{~s}$. These results are the same order of magnitude, but 2-3 $\times$ slower than the same queries we ran on a triple-store implemented directly in Postgres. We attribute this to the fact that we compressed namespace strings in Postgres more aggressively than Sesame does, and we can interact with the triple-store directly in SQL rather than indirectly through Sesame's interfaces and SeRQL. We observed similar results when using Jena instead of Sesame.

Thus, in this paper, we report triple-store numbers using the direct Postgres representation, since this seems to be a more fair comparison to the alternative techniques we explore (where we also directly interact with the database) and allows us to report numbers for aggregation queries. 
Our Postgres implementation of the triple-store contains three columns, one each for subject, property, and object. The table contains three $\mathrm{B}+$ tree indices: one clustered on (subject, property, object), two unclustered on (property, object, subject) and (object, subject, property). We experimentally determined these to be the best performing indices for our query workload. We also maintain the list of the 28 interesting properties described in Sect. 5.3 in a small separate table. The total storage needs for this implementation is 8.3 GBytes (including indices and the dictionary encoding table).

\subsubsection{Property table store}

We implemented clustered property tables as described in Sect. 2.1. To measure their best-case performance, we created a property table for each query containing only the columns accessed by that query. Thus, the table for Q2, Q3, Q4, and Q6 contains the 28 interesting properties described in Sect. 5.3. The table for Q1 stores only subject and Type property columns, allowing for repetitions in the subject for multi-valued attributes. The table for Q5 contains columns for subject, Origin, Records, and Type. The Q7 table contains subject, Encoding, Point, and Type columns. We will look at the performance consequences of property tables that are wider than needed to answer particular queries in Sect. 6.7.

For all but Q1, multi-valued attributes are stored in columns that are integer arrays (int[] in Postgres), while all other columns are integer types. For single-valued attributes that are used as selection predicates, we create unclustered $\mathrm{B}+$ tree indices. We attempted to use GiST [25] indexing for integer arrays in Postgres, ${ }^{2}$ but using this access path took more time than a sequential scan through the database, so multi-valued attributes used as selection predicates were not indexed. All tables had a clustered index on subject. While the smaller tables took less space, the property table with 28 properties took 14 GBytes (including indices and the dictionary encoding table).

\subsubsection{Vertically partitioned store in Postgres}

The vertically partitioned store contains one table per property. Each table contains a subject and object column. There is a clustered $\mathrm{B}+$ tree index on subject, and an unclustered $\mathrm{B}+$ tree index on object. Multi-valued attributes are represented as described in Sect. 3.1 through multiple rows in the table with the same subject and different object value. This store took up 5.2 GBytes (including indices and the dictionary encoding table).

\footnotetext{
$\overline{2}$ http://www.sai.msu.su/ megera/postgres/gist/intarray/README. intarray.
}

\subsubsection{Column-oriented store}

Properties are stored on disk in separate files, in blocks of 64 KB. Each property contains two columns like the vertically partitioned store above. Each property has a clustered B+ tree on subject; and single-valued, low cardinality properties have a bit-map index on object. We used the C-Store default of $4 \mathrm{MB}$ column prefetching (this reduces seeks in merge joins). This store took up 2.7 GBytes (including indices and the dictionary encoding table).

\subsection{Query implementation details}

In this section, we discuss the implementation of all seven benchmark queries in the four designs described above.

Q1. On a triple-store, Q1 does not require a join, and aggregation can occur directly on the object column after the property=Type selection is performed. The vertically partitioned table and the column-store aggregate the object values for the Type table. Because the property table solution has the same schema as the vertically partitioned table for this query, the query plan is the same.

Q2. On a triple-store, this query requires a selection on property=Type and object=Text, followed by a self-join on subject to find what other properties are defined for these subjects. The final step is an aggregation over the properties of the newly joined triples table. In the property table solution, the selection predicate Type $=$ Text is applied, and then the counts of the non-NULL values for each of the 28 columns is written to a temporary table. The counts are then selected out of the temporary table and unioned together to produce the correct results schema. The vertically partitioned store and column-store select the subjects for which the Type table has object value Text, and store these in a temporary table, $t$. They then union the results of joining each property's table with $t$ and count all elements of the resulting joins.

Q3. On a triple-store, Q3 requires the same selection and self-join on subject as Q2. However, the aggregation groups by both property and object value.

The property table store applies the selection predicate Type $=$ Text as in $\mathrm{Q} 2$, but is unable to perform the aggregation on all columns in a single scan of the property table. This is because grouping must be per property and then object for each column, and thus each column must group by the object values in that particular column (a single GROUP BY clause is not sufficient). The SQL standard describes GROUP BY GROUPING SETS to allow multiple GROUP BY aggregation groups to be performed in a single sequential scan of a table. Postgres does not implement this feature, and so our query plan requires a sequential scan of the table for each property aggregation (28 sequential scans), which should 
prove to be expensive. There is no way for us to accurately predict how the use of grouping sets would improve performance, but it should greatly reduce the number of sequential scans.

The vertical store and the column store work like they did in Q2, but perform a GROUP BY on the object column of each property after merge joining with the subject temporary table. They then union together the aggregated results from each property.

Q4. On a triple-store, $\mathrm{Q} 4$ has a selection for property $=$ Language and object=French at the bottom of the query plan. This selection is joined with the Type Text selection (again a self-join on subject), before a second self-join on subject is performed to find the other properties and objects defined for this refined subject list.

The property table store performs exactly as it did in Q3, but adds an extra selection predicate on Language $=$ French .

The vertically partitioned and column stores work as they did in Q3, except that the temporary table of subjects is further narrowed down by a join with subjects whose Language table has object=French.

Q5. On a triple-store, this requires a selection on property= Origin and object $=D L C$, followed by a self-join on subject to extract the other properties of these subjects. For those subjects with the Records property defined, we do a subjectobject join to get the types of the subjects that were objects of the Records property.

For the property table approach, a selection predicate is applied on Origin $=D L C$, and the Records column of the resulting tuples is projected and (self) joined with the subject column of the original property table. The type values of the join results are extracted.

On the vertically partitioned and column stores, we perform the object $=D L C$ selection on the Origin property, join these subjects with the Records table, and perform a subjectobject join on the Records objects with the Type subjects to attain the inferred types.

Note that as described in Sect. 4, subject-object joins are slower than subject-subject joins because the object column is not sorted in any of the approaches. We discuss how the materialized path expression optimization described in Sect. 4 affects the results of this query and Q6 in Sect. 6.6.

Q6. On a triple-store, the query first finds subjects that are directly of Type: Text through a simple selection predicate, and then finds subjects that are inferred to be of Type Text by performing a subject-object join through the records property as in Q5. Next, it finds the other properties defined on this working set of subjects through a self-join on subject. Finally, it performs a count aggregation on these defined properties.

The property table, vertical partitioning, and column-store approaches first create temporary tables by the methods of

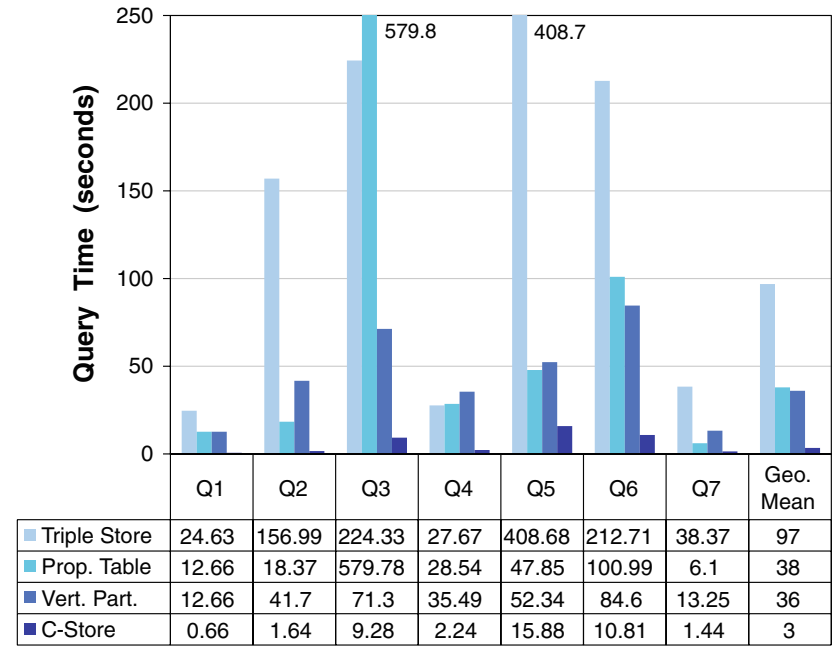

Fig. 4 Performance comparison of the triple-store schema with the property table and vertically partitioned schemas (all three implemented in Postgres) and with the vertically partitioned schema implemented in C-Store. Property tables contain only the columns necessary to execute a particular query

Q2 and Q5, and perform aggregation in a similar fashion to Q2.

Q7. To implement Q7 on a triple-store, the selection on the Point property is performed, and then two self-joins are performed to extract the Encoding and Type values for the subjects that passed the predicate.

In the property table schema, the property table is narrowed down by a filter on Point, which is accessed by an index. At this point, the other three columns (subject, Encoding, Type) are projected out of the table.

In the vertically partitioned and column-store approaches, we join the filtered Point table's subject with those of the Encoding and Type tables, returning the result.

Since this query returns slightly less than 75,000 triples, we avoid the final join with the string dictionary table for this query since this would dominate query time and is the same for all four approaches. We are exploring intelligent caching techniques to reduce the cost of this final dictionary decoding step for high cardinality queries.

\subsection{Results}

The performance numbers for all seven queries on the four architectures are shown in Fig. 4. All times presented in this paper are the average of three runs of the queries. Between queries we copy a 2 GByte file to clear the operating system cache, and restart the database to clear any internal caches.

The property table and vertical partitioning approaches both perform a factor of 2-3 faster than the triple-store 
approach (the geometric mean ${ }^{3}$ of their query times was 38 and $36 \mathrm{~s}$, respectively, compared with $97 \mathrm{~s}$ for the triple-store approach. ${ }^{4} \mathrm{C}$-Store added another factor of 10 performance improvement with a geometric mean of $3 \mathrm{~s}$ (and so is a factor of 32 faster than the triple-store).

To better understand the reasons for the differences in performance between approaches, we look at the performance differences for each query. For Q1, the property table and vertical partitioning numbers are identical because we use the idealized property table for each query, and since this query only accesses one property, the idealized property table is identical to the vertically partitioned table. The triple-store only performs a factor of two slower since it does not have to perform any joins for this query. Perhaps surprisingly, $\mathrm{C}$-Store performs an order of magnitude better. To understand why, we broke the query down into pieces. First, we noted that the type property table in Postgres takes $472 \mathrm{MB}$ compared to just $100 \mathrm{MB}$ in C-Store. This is almost entirely due to the fact that the Postgres tuple header is 27 bytes compared with just 8 bytes of actual data per tuple and so the Postgres table scan needs to read 35 bytes per tuple (actually, more than this if one includes the pointer to the tuple in the page header) compared with just 8 for C-Store.

Another reason why C-Store performs better is that it uses an index nested loops join to join keys with the strings dictionary table while Postgres chooses to do a merge join. This final join takes $5 \mathrm{~s}$ longer in Postgres than it does in C-Store (this $5 \mathrm{~s}$ overhead is observable in the other queries as well). These two reasons account for the majority of the performance difference between the systems; however, the other advantages of using a column-store described in Sect. 3.2 are also a factor.

Q2 shows why avoiding the expensive subject-subject joins of the triple-store is crucial, since the triple-store performs much more slowly than the other systems. The vertical partitioning approach is outperformed by the property table approach since it performs 28 merge joins that the property table approach does not need to do (again, the property table approach is helped by the fact that we use the optimal property table for each query).

As expected, the multiple sequential scans of the property table hurt it in Q3. Q4 is so highly selective that the query results for all but C-Store are quite similar. The results of the optimal property table in Q5-Q7 are on par with those of the vertically partitioned option, and show that subject-object joins hurt each of the stores significantly.

\footnotetext{
${ }^{3}$ We use geometric mean - the $n$th root of the product of $n$ numbersinstead of the arithmetic mean since it provides a more accurate reflection of the total speedup factor.

${ }^{4}$ If we hand-optimized the triple-store query plans rather than use the Postgres default, we were able reduce the mean to $79 \mathrm{~s}$; this demonstrates the fact that by introducing a number of self-joins, queries over a triple-store schema are very hard to optimize.
}

On the whole, vertically partitioning a database provides a significant performance improvement over the triple-store schema, and performs similarly to property tables. Given that vertical partitioning in a row-oriented database is competitive with the optimal scenario for a property table solution, we conclude that they are the preferable solution since they are simpler to implement. Further, if one uses a database designed for vertically partitioned data such as $\mathrm{C}$-Store, additional performance improvement can be realized. C-Store achieved nearly interactive time on our benchmark running on a single machine that is 2 years old.

We also note that multi-valued attributes play a role in reducing the performance of the property table approach. Because we implement multi-valued attributes in property tables as arrays, simple indexing can not be performed on these arrays, and the GiST [25] indexing of integer arrays performs worse than a sequential scan of the property table.

Finally, we remind the reader that the property tables for each query are idealized in that they only include the subset of columns that are required for the query. As we will show in Sect. 6.7, poor choice in columns for a property table will lead to less-than-optimal results, whereas the vertical partitioning solution represents the best- and worst-case scenarios for all queries.

\subsubsection{Postgres as a choice of RDBMS}

There are several notes to consider that apply to our choice of Postgres as the RDBMS. First, for Q3 and Q4, performance for the property table approach would be improved if Postgres implemented GROUP BY GROUPING SETs.

Second, for the vertically partitioned schema, Postgres processes subject-subject joins non-optimally. For queries that feature the creation of a temporary table containing subjects that are to be joined with the subjects of the other properties' tables, we know that the temporary list of subjects will be in sorted order, as it comes from a table that is clustered on subject. Postgres does not carry this information into the temporary table, and will only perform a merge join for intermediate tuples that are guaranteed to be sorted. To simulate the fact that other databases would maintain the metadata about the sorted temporary subject list, we create a clustered index on the temporary table before the UNIONJOIN operation. We only included the time to create the temporary table and the UNION-JOIN operations in the total query time, as the clustering is a Postgres implementation artifact.

Further, Postgres does not assume that a table clustered on an attribute is in perfectly sorted order (due to possible modifications after the cluster operation), and thus can not perform the merge join directly; rather it does so in conjunction with an index scan, as the index is in sorted order. This process incurs extra seeks as the leaves of the $\mathrm{B}+$ tree are 


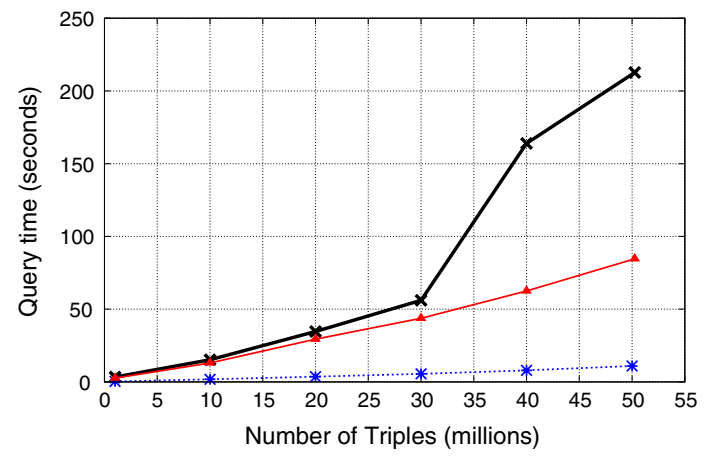

Triple Store $\boldsymbol{*}$ - C-Store …*... Vertical Partitioning $-\_$

Fig. 5 Query 6 performance as number of triples scale

traversed, leading to a significant cost effect compared to the inexpensive merge join operations of C-Store.

With a different choice of RDBMS, performance results might differ, but we remain convinced that Postgres was a good choice of RDBMS, given that it handles NULL values so well, and thus enabled us to fairly benchmark the property table solutions.

\subsection{Scalability}

Although the magnitude of query performance is important, an arguably more important factor to consider is how performance scales with size of data. In order to determine this, we varied the number of triples we used from the library dataset from one million to fifty million (we randomly chose what triples to use from a uniform distribution) and reran the benchmark queries. Figure 5 shows the results of this experiment for query 6. Both vertical partitioning schemes (Postgres and C-Store) scale linearly, while the triple-store scales super-linearly. This is because all joins for this query are linear for the vertically partitioned schemes (either merge joins for the subject-subject joins, or index scan merge joins for the subject-object inference step); however the triple-store sorts the intermediate results after performing the three selections and before performing the merge join. We observed similar results for all queries except queries 1, 4, and 7 (where the triple-store also scales linearly, but with a much higher slope relative to the vertically partitioned schemes).

\subsection{Materialized path expressions}

As described in Sect. 4, materialized path expressions can remove the need to perform expensive subject-object joins by adding additional columns to the property table or adding an extra table to the vertically partitioned and columnoriented solutions. This makes it possible to replace subjectobject joins with cheaper subject-subject joins. Since Queries 5 and 6 contain subject-object joins, we reran just those
Table 2 Query times (in s) for Q5 and Q6 after the Records:Type path is materialized. $\%$ faster $=\frac{100 \mid \text { original }- \text { new } \mid}{\text { original }}$

\begin{tabular}{lll}
\hline & Q5 & Q6 \\
\hline Property table & $39.49(17.5 \%$ faster $)$ & $62.6(38 \%$ faster $)$ \\
Vertical partitioning & $4.42(92 \%$ faster $)$ & $65.84(22 \%$ faster $)$ \\
C-Store & $2.57(84 \%$ faster $)$ & $2.70(75 \%$ faster $)$ \\
\hline
\end{tabular}

experiments using materialized path expressions. Recall that in these queries we join object values from the Records property with subject values to get those subjects that can be inferred to be a particular type through the Records property.

For the property table approach, we widened the property table by adding a new column representing the materialized path expression: Records:Type. This column indicates the type of entity that is related to a subject through the Records property (if a subject does not have a Records property defined, its value in this column will be NULL). Similarly, for the vertically partitioned and column-oriented solutions, we added a table containing a subject column and a Records:Type object column, thus allowing one to find the Type of objects that a resource Records with a cheap subject-subject merge join. The results are displayed in Table 2.

It is clear that materializing the path expression and removing the subject-object join results in significant improvement for all schemas. However, the vertically partitioned schemas see a greater benefit since the materialized path expression is multi-valued (which is the common case, since if at least one property along the path is multi-valued, then the materialized result will be multi-valued).

In summary, Q5 and Q6, which used to take 400 and $200 \mathrm{~s}$, respectively, on the triple-store, now take less than 3 seconds on the column-store. This represents a two orders of magnitude performance improvement!

\subsection{The effect of further widening}

Given that semantic web content is likely to have an unstructured schema, clustering algorithms will not always yield property tables that are the perfect width for all queries. We now experimentally demonstrate the effect of property tables that are wider than they need to be for the same queries run in the experiments above. Row-stores traditionally perform poorly relative to vertically partitioned schemas and columnstores when queries need to access only a few columns of a wide table, so we expect the performance of the property table implementation to degrade with increasing table width. To measure this, we synthetically added 60 non-sparse random integer-valued columns to the end of each tuple in the widest property table in Postgres. This resulted in an approximately 7 GByte increase in database size. We then reran 
Table 3 Query times (in s) comparing a wider than necessary property table to the property table containing only the columns required for the query

\begin{tabular}{lrc}
\hline Query & Wide property table & $\begin{array}{c}\text { Property table } \\
\text { (\% slowdown) }\end{array}$ \\
\hline Q1 & 60.91 & 381 \\
Q2 & 33.93 & 85 \\
Q3 & 584.84 & 1 \\
Q4 & 44.96 & 58 \\
Q5 & 76.34 & 60 \\
Q6 & 154.33 & 53 \\
Q7 & 24.25 & 298 \\
\hline
\end{tabular}

$\%$ Slowdown $=\frac{100 \mid \text { original-new } \mid}{\text { original }}$. Vertically partitioned stores are not affected

Q1-Q7 on this wide property table. The results are shown in Table 3.

Since each of the queries (except query 1) executes in two parts, first creating a temporary table containing the subset of the relevant data for that query, and then executing the rest of the query on this subset, we see some variance in \% slowdown numbers, where smaller slowdown numbers indicate that a majority of the query time was spent on the second stage of query processing. However, each query sees some degree of slowdown. These results support the notion that while property tables can sometimes outperform vertical partitioning on a row-oriented store, a poor choice of property table can result in significantly poorer query performance. The vertically partitioned solutions are impervious to such effects.

\section{SW-Store Design}

Inspired by our observations from the previous section that column-stores present some clear advantages in storing RDF data, we have begun an initial implementation of an RDF database, called SW-Store. Our hope is that SW-Store will both be able to function as a stand-alone RDF database and alternatively as a storage layer for existing RDF applications such as Jena, Sesame, or the Web-based RDF browser described in Sect. 5.

\subsection{System architecture}

The basic architecture of SW-Store is shown in Fig. 6. The system consists of four major components: a vertically partitioned storage system, a relational query engine that executes queries over those partitions, a query rewriter that converts queries in SQL or SPARQL over RDF triples into queries over the vertically partitioned schema, and a batch-writer

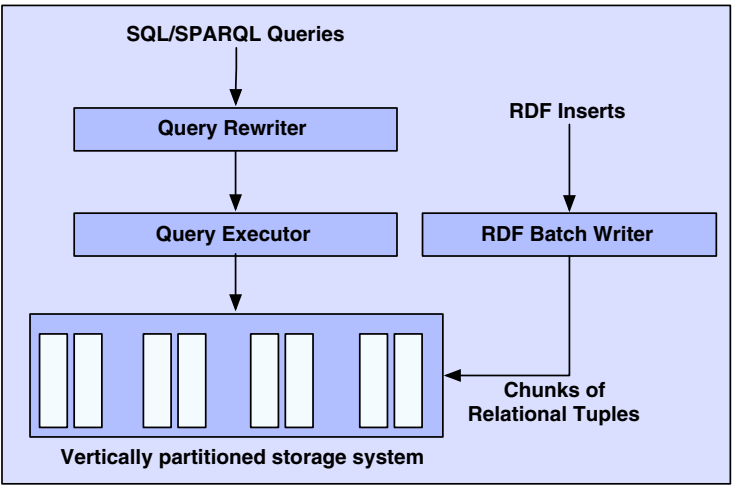

Fig. 6 The architecture of SW-Store

that periodically converts RDF triples stored in an overflow table into vertically partitioned data. We discuss each of these components in more detail in the sections below.

\subsection{Storage system}

As described in Sect. 3, the majority of the data in SW-Store are stored in two-column tables; one table for each unique property in the data set. Each table is sorted by the first (subject) column to facilitate high performance table merge operations.

The default C-Store implementation stores each column of these two-column tables in a separate file, stored on disk in blocks of $64 \mathrm{~KB}$. However, we have found this "pure" column-oriented storage to be non-optimal since most property table accesses require data from both columns. Thus, SW-Store adopts a hybrid storage representation along the lines of PAX [8], where a single block contains data from both columns; however, within a block, data from each column are kept separate.

Blocks may be compressed, and each column within a block can use a different compression method (e.g., runlength, bit-vector, or Lempel-Ziv encoding), as in C-Store [4]. Each block is compressed separately, which allows us to avoid decompressing a whole table to access a few rows.

A single-column table that contains all subjects that appear in at least one vertical partition is also stored. This allows SWStore to avoid storing the subject column for some properties (i.e., the vertical partition becomes a single-column list of object values). In such a situation, there must be a one-to-one mapping between entries in the values column and subjects stored in the subjects table. As shown in Fig. 7b, for a partition representing property $p, \mathrm{SW}-$ Store must store a NULL in the values list for any subject that does not have property $p$. In such cases, the vertical partitioning advantage of not having to store NULL values no longer exists. Of course, NULL data can be compressed, and when the compressed singlecolumn table with NULL values is smaller than the two- 
(a) Original vertically partitioned schema from Section 3.

\begin{tabular}{|c|c|}
\multicolumn{2}{c}{ Type } \\
\hline ID1 & BookType \\
\hline ID2 & CDType \\
\hline ID3 & BookType \\
\hline ID4 & DVDType \\
\hline ID5 & CDType \\
\hline ID6 & BookType \\
\hline
\end{tabular}

\begin{tabular}{|c|c|}
\hline \multicolumn{2}{|c|}{ Title } \\
\hline ID1 & "XYZ" \\
\hline ID2 & "ABC" \\
\hline ID3 & "MNO" \\
\hline ID4 & "DEF" \\
\hline ID5 & "GHI" \\
\hline
\end{tabular}

Copyright
\begin{tabular}{|l|c|}
\hline ID1 & "2001" \\
\hline ID2 & "1985" \\
\hline ID5 & "1995" \\
\hline ID6 & "2004" \\
\hline
\end{tabular}

\begin{tabular}{|c|c|c|c|}
\hline \multicolumn{2}{|c|}{ Author } & \multicolumn{2}{|r|}{ Artist } \\
\hline ID1 & "Fox, Joe" & ID2 & "Orr, Tim" \\
\hline & \multicolumn{2}{|c|}{ Language } & \\
\hline & ID2 & "French" & \\
\hline & ID3 & "English" & \\
\hline
\end{tabular}

(b) The subject column does not need to be stored if object values map one-to-one to an all-subjects table and NULLs used for undefined subject-property combinations. The same data as in (a) is shown below, with the subject columns from each partition removed.
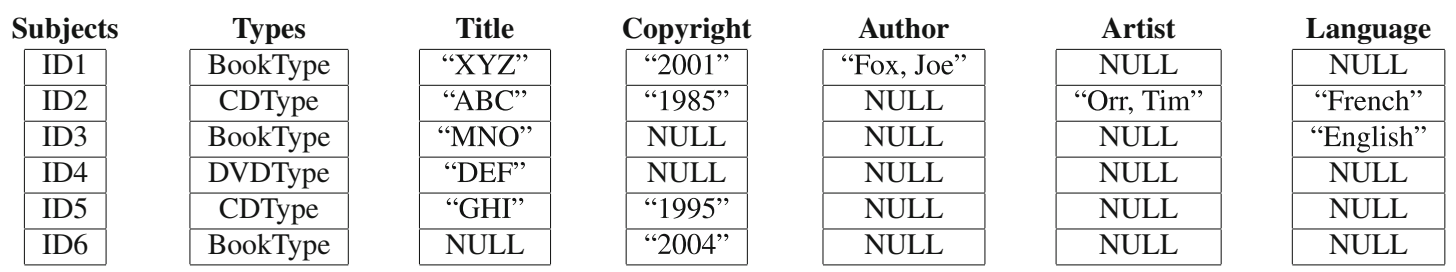

(c) The same schema as in (b) with NULLs being compressed using each of the three types of sparse data compression described in Section 7.2.1.
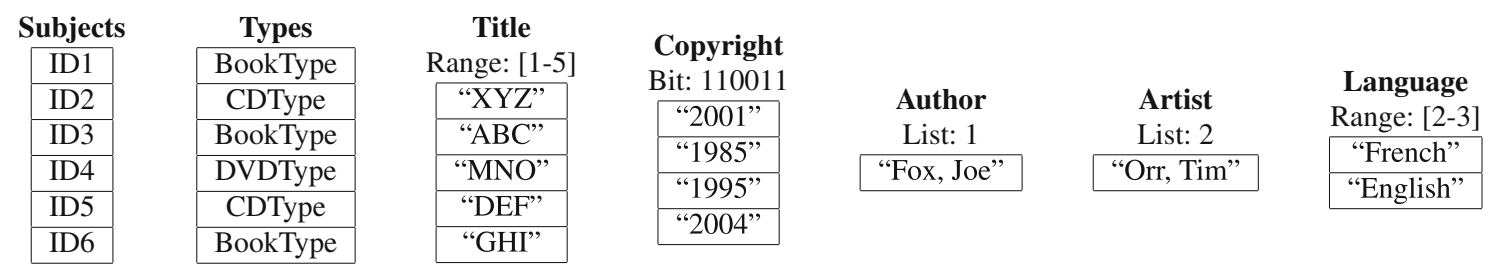

Fig. 7 Vertically partitioned storage system

column table without NULL values, SW-Store chooses the single-column representation. We describe several compression methods we use for sparse (NULL-rich) data in Sect. 7.2.1 below. In the current implementation, multi-valued properties cannot be implemented as single column tables (due to the need for one-to-one value-to-subject mapping); however, we envision dropping this requirement in the future (a special flag indicating that the current value is actually multiple values, followed by the number of values and then the values themselves could be used).

Both columns from each vertical partition can be indexed, as appropriate. Since the first column is always sorted in each vertical partition, a sparse index is used to index this column. Further, an inverted index on all string data can be maintained to improve performance of string-matching queries.

\subsubsection{Representing sparse data}

As described above, in some cases it is preferable to avoid storing the subject column in the vertical partition for a property $p$, instead storing just one value in the vertical partition for each possible subject, with a NULL value stored if the subject does not have property $p$. In this case, it is usually desirable to aggressively compress the NULL data. We follow the column-oriented sparse compression schemes presented in our previous work [2]. Sparse data is stored using three different encodings for NULLs. For very dense data or for data where NULLs come in long runs, we store raw data along with tupleID ranges indicating which tupleIDs are non-NULL (where a tupleID is the ordinal offset of a value in the single-column table listing all subjects in the dataset). For example, if tupleID 7 is the only NULL element in a ten-tuple column, the column would list the nine nonNULL elements in order, followed by the ranges $[1,6]$ and $[8,10]$ indicating the ranges of the non-NULL tupleIDs. In the worst case, where no two NULLs occur consecutively, the overhead is two integer tupleIDs per NULL element. For dense data, this overhead is small. An example of this type of encoding is presented in Fig. 7c for the "Title" and "Language" partitions.

For data that are neither dense nor sparse, we store the raw non-NULL data for property $p$ along with a bitmap with one bit per subject. A one at offset $i$ in the bitmap indicates that subject $i$ defines the particular $p$ (i.e., it has a non-NULL value in the vertical partition for $p$ ). For example if every alternative element is NULL in a vertical partition that maps 
to a ten-value subject table, each non-NULL element would be stored in order, followed by the bitmap 1010101010. The overhead is one-bit per subject in the dataset (regardless of the column density). An example of this type of encoding is presented in Fig. 7c for the "Copyright" partition.

For data that are sparse, we store the raw non-NULL data along with a list of tupleIDs of the non-NULL elements. So if tupleID 7 is the only non-NULL element in a ten-tuple column, the column would consist of a single value (the value of tuple 7), along with a single tupleID (7). The overhead is one integer tupleID per non-NULL element; but for sparse datasets this overhead is small. An example of this type of encoding is presented in Fig. 7c for the "Author" and "Artist" partitions. Note that when subjects are encoded using integer keys, this technique is identical to the two-column vertical partitioned representation.

Our previous work [2] shows experimentally that for data of any sparsity, as long as the correct compression technique described above is used, the overhead of the NULL elements is small.

\subsection{Query engine}

Querying a collection of tables requires query plans to merge results from individual tables together. A typical plan for accessing a column store is shown in Fig. 8. Here, each column is scanned independently to produce a list of tuple positions that satisfy all three predicates; those positions are then fed into a "tupleize" operator that combines the three columns into a relational tuple for output to the user.

Plans in SW-Store work similarly, where columns in a column-store map to the two-column vertical partitions in SW-Store, a position in a column-store maps to the subject column in the two-column vertical partitions, and "tupleize" in a column-store becomes "merge-join" over the twocolumn vertical partitions. Since two-column tables in SW-Store are stored as a single hybrid column, it was straight-

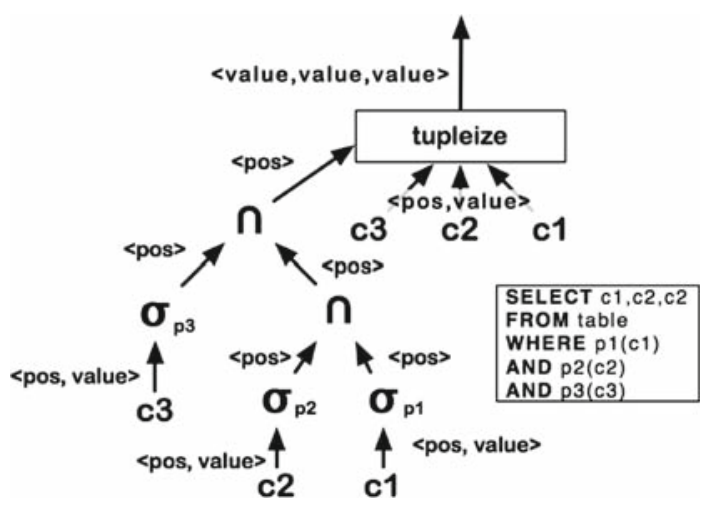

Fig. 8 A column-oriented SW-Store query plan forward to extend the C-Store column-oriented operators to take data from these hybrid columns.

In general, the tupleize (merge-join) operator can be performed at any point in the query plan. For example, the plan in Fig. 8 could be rewritten so that each column (partition) is merged at the beginning of the plan, before the predicates are applied. SW-Store uses the heuristics presented in our previous work on column-oriented tuple materialization [6] to make this decision. In most cases, it is best to perform the merge-join as late as possible, under the well-known "push selections past joins" database optimization rule. For plans that contain an aggregation operator, merge-join can also be deferred until after the aggregate, since typically only a subset of the properties are involved in the aggregation. However, for plans that involve subject-object joins, a decision must be made as to whether to place the merge-join operator before the subject-object join operators. This decision is generally based on the expected cardinality of the join $[5,6]$. In most cases, SW-Store performs the merge-joins before the subject-object joins.

There is, however, an additional complication for columnoriented query plans, since operators in these query plans no longer form trees as in most database systems. In fact, it is very common for an operator to have multiple parents. For example, as shown in Fig. 9, if a predicate is applied to a vertical partition, the result of the predicate can be represented as a list of subjects whose object values for the property (that this vertical partition corresponds to) pass the predicate. This list of subjects might need to be sent to multiple other vertical partitions (corresponding to subject-object values for other properties) to extract the object values at this list of subjects. These object extraction operators thus serve as multiple parents for the selection operator, turning the query plan into a graph of operators rather than a tree.

This lack of tree structure is problematic for a pull-based iterator model. If one parent consumes its input data at a faster rate than the other, data might be lost before the slower parent has an opportunity to process it. The problem is solved by ensuring that the graph is still rooted with a single node that has no parents. As long as this node requests data at the same rate from all of its children (and this property holds recursively for every other descendant node in the graph), then nodes with more than one parent are guaranteed not to have the problem of one parent requesting data at a faster rate than the other. Thus, operator code is written very carefullywhen an operator's own "getNextBlock" is called, it will call "getNextBlock" on a child operator only if all data from the previous block have been processed and are guaranteed never to be needed again, and the last position (corresponding to a subject value) processed from the previous block is equal to the last position processed from all of the other children blocks. 
Fig. 9 A non-tree query plan

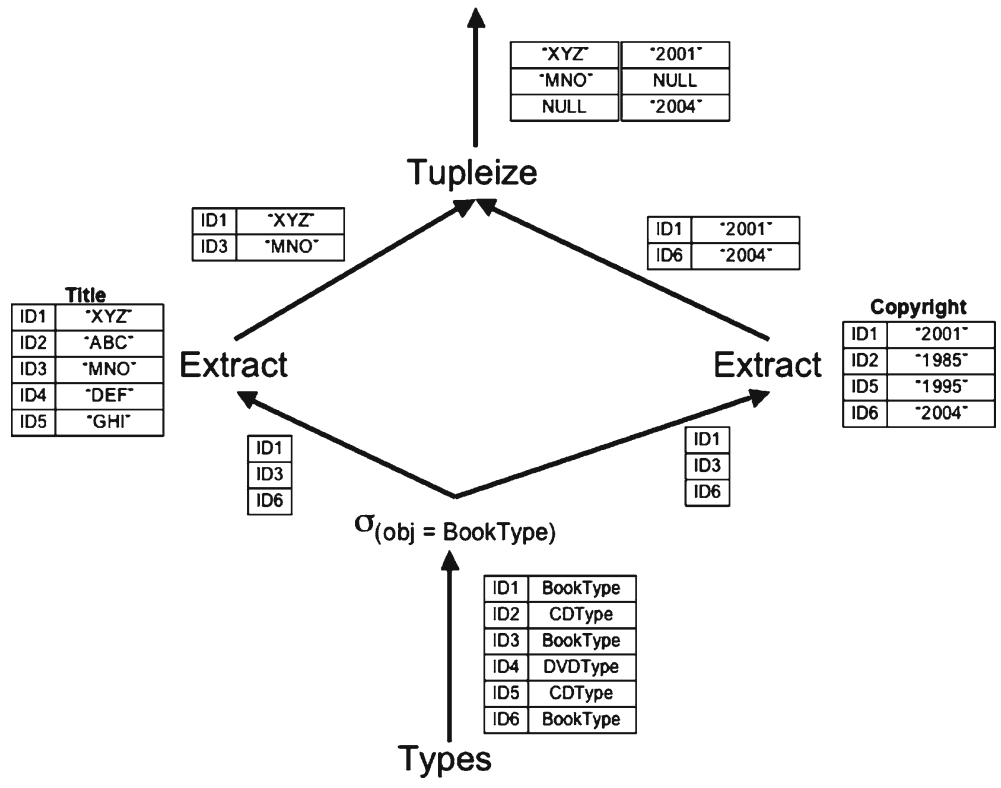

\subsubsection{Overflow tables}

To support inserts of data (as described in Sect. 7.5) without requiring updates to the compressed vertical partitions, SW-Store includes a small overflow table containing triples (stored using the standard triples schema), similar to the overflow tables demonstrated in Table 1c, d. Queries must therefore go both to the triples table and the vertical partitions and a merge must be performed at the top of the query plan. Given that the overflow table is not read-optimized (it is not sorted, indexed, or compressed), we require that the overflow table be kept small —if it exceeds a threshold parameter, some percentage of its data must be removed, and added to the corresponding vertical partitions (see Sect. 7.5). Overflow tables may also turn out to be useful for storing some very rare predicates.

\subsubsection{Materialized joins}

As described in Sect. 4, materializing paths in the RDF as additional vertical partitions can remove subject-object joins and improve performance.

There is, however, a cost in having a larger number of extra materialized vertical partitions, since they need to be recalculated whenever new triples are added to SW-Store. For read-only or read-mostly RDF applications, the number of materialized columns will be larger in SW-Store than for applications where data gets inserted more frequently. In the case where the number of extra vertical partitions is limited, the choice of what joins to materialize is based on prior queries.

\subsection{Query translation}

When a SQL or SPARQL query arrives in SW-Store, it must be converted to a query over the vertical partitions that are in the system. We have written an automatic query translater [10], but at the time of this publication have not yet integrated the translater into the SW-Store, since C-Store's generic plan generator remains rudimentary. This section describes how query translation works.

Queries that arrive in SPARQL, must be converted to both SQL over a triples table (for querying the overflow table, Sect. 7.3.1), and SQL over a vertically partitioned store. An important goal is to push as much of the SPARQL graph filtering as possible into the SQL [30]. We use Jena's ARQ module for SPARQL query parsing and implement our own internal representation for resulting query objects. This internal representation is then converted to SQL over the triple store and vertically partitioned schemas.

For SQL conversion with the vertically partitioned schema, each filter over a property (e.g., "property = 'pubyear' AND object $=$ '2005" or "property = 'type' AND object $=$ "foo"') is converted to a filter over the corresponding vertical partition (e.g., "pubyear.object $=$ '2005"').

We illustrate this process through an example. The following query over a RDF triples table finds the birthday of the author of the book "The History of VLDBJ":

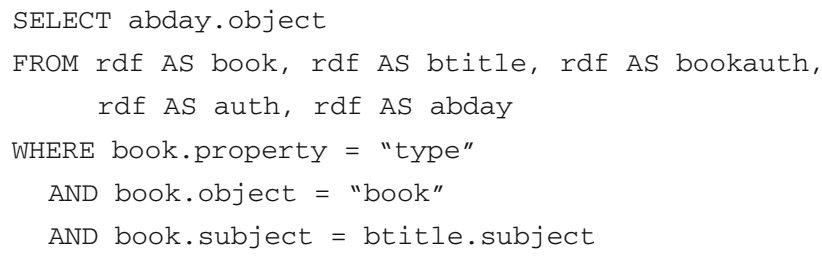




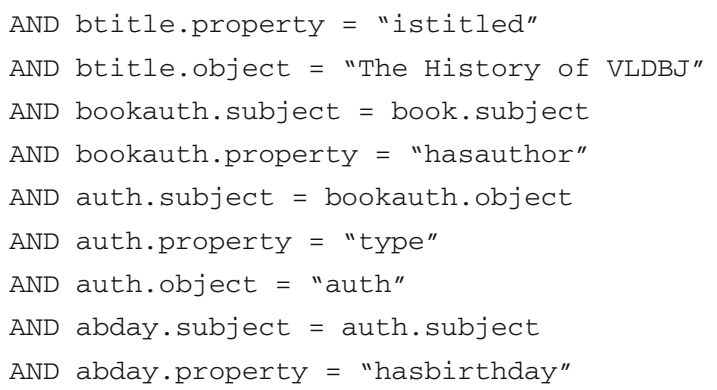

Over a vertically partitioned schema, this becomes:

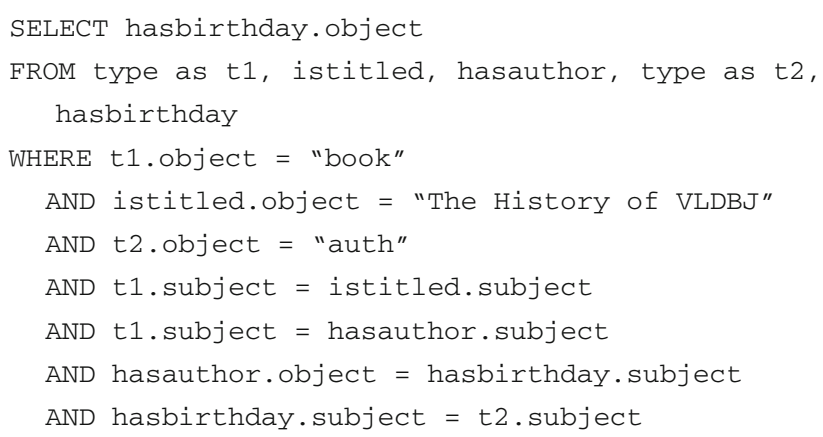

Here, all joins are fast subject-subject joins except the hasauthor-hasbirthday join, because we have not materialized the "author $\rightarrow$ birthday" path expression.

\subsection{Updates}

As in any vertically partitioned store, adding data to SW-Store is potentially expensive as each update requires writing to many columns. As in other column-store systems [45], we address this issue by batching writes. In SWStore, these updates can simply be added to the overflow table, which the query executor must already merge into query results.

A background merge process periodically scans the overflow table to determine whether data need to be moved into vertical partitions. It decides to move data from the overflow table if the size of the overflow table exceeds a threshold, so that the cost of scanning the overflow table relative to accessing the read optimized vertical partitions at query time (see Sect. 7.3.1) remains small.

Merging data from the overflow table into the vertical partitions can be expensive since the vertical partitions need to be decompressed before the merge and re-compressed and re-indexed afterwards. We keep the cost of merging low in two ways. First, not all triples are moved out of the overflow table upon a merge. Triples containing properties that only appear a small number of times in the overflow table are not merged into the corresponding vertical partitions since the relative cost of merging these small number of triples outweighs the benefit of removing them from the overflow table.

Second, we partition each vertical partition horizontally into "chunks", such that each chunk represents a collection of data inserted over a particular time period. When data intended for a particular vertical partition is initially moved out of the overflow table, it is not immediately merged into any of these previously existing chunks; rather a new chunk is created containing just this new data. Each additional chunk created by this process, however, increases query time, as queries must be run over all chunks, and each chunk must be compressed and encoded separately. Hence, a different background process merges small chunks into larger ones. This process dramatically increases the efficiency of merging data from the overflow table. Instead of decompressing and re-compressing the entire vertical partition every time data are added, only small subsets of the vertical partition are merged with the new data, and the cost of decompressing and re-compressing is amortized over larger chunk merges.

\section{Conclusion}

The emergence of the Semantic Web necessitates highperformance data management tools to manage the tremendous collections of RDF data being produced. Current state of the art RDF databases-triple-stores-scale extremely poorly since most queries require multiple self-joins on the triples table. The previously proposed "property table" optimization has not been adopted in most RDF databases, perhaps due to its complexity and inability to handle multivalued attributes. We showed that a poorly selected property table can result in significant performance degradation over an optimal property table, thus making the solution difficult to use in practice (in our experiments we saw up to $381 \%$ slowdown). As an alternative to property tables, we proposed vertically partitioning tables and demonstrated that they achieve similar performance as property tables in a row-oriented database, while being simpler to implement. Further, we showed that on a version of the C-Store column-oriented database, it is possible to achieve a factor of 32 performance improvement over the current state of the art triple store design. Queries that used to take hundreds of seconds can now be run in less than 10 seconds, a significant step toward interactive-time semantic web content storage and querying. These results illustrate the need for a DBMS designed specifically for RDF data management, and this is the motivation of the SW-Store database project.

Acknowledgments We thank George Huo and the Postgres development team for their advice on our Postgres implementation, and Michael Stonebraker for his feedback on this paper. This work was supported by the National Science Foundation under grants IIS-048124, CNS0520032, IIS-0325703, and IIS-0845643. 


\section{Appendix}

Below are the seven benchmark queries as implemented on a triple-store. Note that for clarity of presentation, the predicates are shown on raw strings (instead of the dictionary encoded values) and the post-processing dictionary decoding step is not shown. Further, URIs have been abbreviated (the full URIs and the list of 28 properties in the properties table are presented in our technical report [1]).

\section{Query1:}

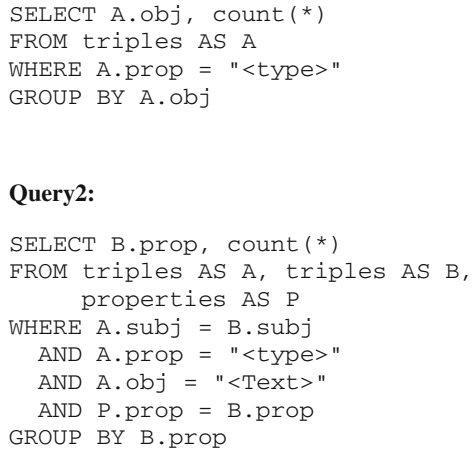

Query2:

\section{Query3:}

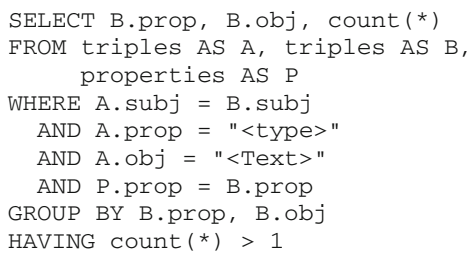

\section{Query4:}

SELECT B.prop, B.obj, count (*)

FROM triples AS A,

\section{Query5:}

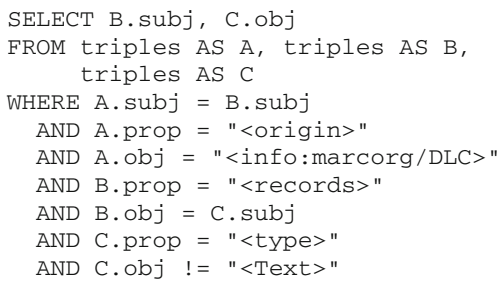

Query6:

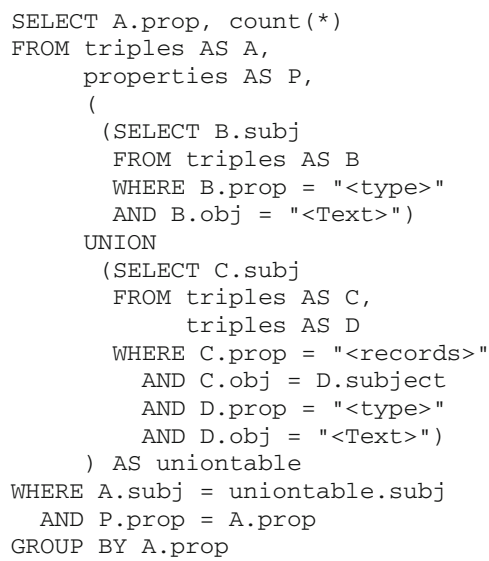

Query7:

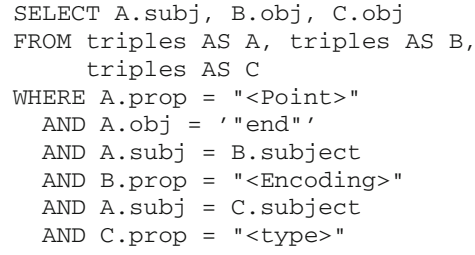

\section{References}

1. Abadi, D., Marcus, A., Madden, S., Hollenbach, K.: Using the Barton libraries dataset as an RDF benchmark. Technical Report MIT-CSAIL-TR-2007-036, MIT Press, USA

2. Abadi, D.J.: Column stores for wide and sparse data. In: CIDR (2007)

3. Abadi, D.J.: Query execution in column-oriented database systems. PhD Dissertation, PhD Thesis, MIT Press, USA (2008)

4. Abadi, D.J., Madden, S., Ferreira, M.: Integrating compression and execution in column-oriented database systems. In: SIGMOD (2006)

5. Abadi, D.J., Madden, S.R., Hachem, N.: Column-stores vs. rowstores: How different are they really? In: SIGMOD (2008)

6. Abadi, D.J., Myers, D.S., DeWitt, D.J., Madden, S.R.: Materialization strategies in a column-oriented DBMS. In: Proceedings of $\operatorname{ICDE}(2007)$

7. Agrawal, R., Somani, A., Xu, Y.: Storage and querying of E-commerce data. In: VLDB (2001)

8. Ailamaki, A., DeWitt, D.J., Hill, M.D., Skounakis, M.: Weaving relations for cache performance. In: VLDB, pp. 169-180 (2001)

9. Alexaki, S., Christophides, V., Karvounarakis, G., Plexousakis, D., Tolle, K.: The ICS-FORTH RDFSuite: managing voluminous RDF description bases. In: SemWeb (2001)

10. Bajda-Pawlikowski, K.: Querying RDF data stored in DBMS SPARQL to SQL Conversion. Technical Report TR-1409, Yale Computer Science Department, USA

11. Batory, D.S.: On searching transposed files. ACM Trans. Database Syst. 4(4), 531-544 (1979)

12. Beckmann, J., Halverson, A., Krishnamurthy, R., Naughton, J.: Extending RDBMSs to support sparse datasets using an interpreted attribute storage format. In: ICDE (2006)

13. Bertino, E., Kim, W.: Indexing techniques for queries on nested objects. IEEE Trans. Knowl. Data Eng. 1(2), 196-214 (1989) 
14. Boncz, P., Manegold, S., Kersten, M.: Database architecture optimized for the new bottleneck: memory access. In: VLDB, pp. 54-65 (1999)

15. Boncz, P.A., Kersten, M.L.: MIL primitives for querying a fragmented world. VLDB J. 8(2), 101-119 (1999)

16. Boncz, P.A., Zukowski, M., Nes, N.: MonetDB/X100: hyperpipelining query execution. In: CIDR, pp. 225-237 (2005)

17. Bonstrom, V., Hinze, A., Schweppe, H.: Storing RDF as a graph. In: Proceedings of LA-WEB (2003)

18. Broekstra, J., Kampman, A., van Harmelen, F.: Sesame: a generic architecture for storing and querying RDF and RDF schema. In: ISWC, pp. 54-68 (2002)

19. Chong, E.I., Das, S., Eadon, G., Srinivasan, J.: An efficient SQLbased RDF querying scheme. In: VLDB, pp. 1216-1227 (2005)

20. Copeland, G.P., Khoshafian, S.N.: A decomposition storage model. In: Proceedings of SIGMOD, pp. 268-279 (1985)

21. Corwin, J., Silberschatz, A., Miller, P.L., Marenco, L.: Dynamic tables: An architecture for managing evolving, heterogeneous biomedical data in relational database management systems. J. Am. Med. Inf. Assoc. 14(1), 86-93 (2007)

22. Falcons. Searching the semantic web. Web page. http://iws.seu. edu.cn/services/falcons/objectsearch/index.jsp/

23. Florescu, D., Kossmann, D.: Storing and querying XML data using an RDMBS. IEEE Data Eng. Bull. 22(3), 27-34 (1999)

24. Harris, S., Gibbins, N.: 3store: efficient bulk RDF storage. In: Proceedings of PSSS'03, pp. 1-15 (2003)

25. Hellerstein, J.M., Naughton, J.F., Pfeffer, A.: Generalized search trees for database systems. In: Proceedings of VLDB, pp. 562-573. Zurich (1995)

26. Howe, B., Maier, D., Rayner, N., Rucker, J.: Quarrying dataspaces: schemaless profiling of unfamiliar information sources. In: Proceedings of the workshop on information integration methods, architectures, and systems (IIMAS) (2008)

27. Kemper, A., Moerkotte, G.: Access support relations: an indexing method for object bases. Inf. Syst. 17(2), 117-145 (1992)

28. Library catalog data. http://simile.mit.edu/rdf-test-data/barton/

29. Longwell: http://simile.mit.edu/longwell/

30. Lu, J., Cao, F., Ma, L., Yu, Y., Pan, Y.: An Effective SPARQL support over relational databases. In: Proceedings of the joint ODBIS/SWDB workshop on semantic web, ontologies, and databases (2007)

31. Lu, J., Ma, L., Zhang, L., Brunner, J.-S., Wang, C., Pan, Y., Yu, Y.: SOR: A practical system for ontology storage, reasoning and search. In: Proceedings of VLDB, pp. 1402-1405 (2007)

32. Lu, J., Wang, C., Ma, L., Yu, Y., Pan, Y.: Performance and scalability evaluation of practical ontology systems. In: Proceedings of the joint ODBIS/SWDB workshop on semantic web, ontologies, and databases (2007)
33. MacNicol, R., French, B.: Sybase IQ multiplex-designed for analytics. In: VLDB pp. 1227-1230 (2004)

34. Metaweb: Freebase parallax. Web page. http://mqlx.com/ david/ parallax/

35. Milo, T., Suciu, D.: Index structures for path expressions. In: Proceedings of ICDT, pp. 277-295 (1999)

36. Olofson, C.: Worldwide rdbms 2005 vendor shares. Technical report 201692, IDC, USA (2006)

37. Redland RDF application framework. http://librdf.org/

38. RDF Primer. W3C Recommendation. http://www.w3.org/TR/rdfprimer (2004)

39. RDQL-A Query Language for RDF. W3C Member Submission 9 January 2004. http://www.w3.org/Submission/RDQL/, 2004

40. Simile website. http://simile.mit.edu/

41. SPARQL Query Language for RDF. W3C Working Draft 4 October 2006. http://www.w3.org/TR/rdf-sparql-query/, 2006

42. Schmidt, M., Hornung, T., Kuchlin, N., Lausen, G., Pinkel, C.: An experimental comparison of RDF data management approaches in a SPARQL benchmark scenario. In: Proceedings of ISWC (2008)

43. Shanmugasundaram, J., Tufte, K., Zhang, C., He, G., DeWitt, D.J., Naughton, J.F.: Relational databases for querying XML documents: Limitations and opportunities. In: Proceedings of VLDB, pp. 302-314 (1999)

44. Sindice. The semantic web index. http://sindice.com/

45. Stonebraker, M., Abadi, D.J., Batkin, A., Chen, X., Cherniack, M., Ferreira, M., Lau, E., Lin, A., Madden, S., O’Neil, E.J., O’Neil, P.E., Rasin, A., Tran, N., Zdonik, S.B.: C-Store: a column-oriented DBMS. In: VLDB, pp. 553-564 (2005)

46. Swoogle: Semantic web search engine. http://swoogle.umbc.edu/

47. Theoharis, Y., Christophides, V., Karvounarakis, G.: Benchmarking database representations of RDF/S stores. In: Proceedings of ISWC (2005)

48. UniProt: RDF dataset. http://dev.isb-sib.ch/projects/uniprot-rdf/

49. Vesset, D.: Worldwide data warehousing tools 2005 vendor shares. Technical report 203229, IDC, USA (2006)

50. W3C SWEO Community Project: Linking open data on the semantic web. http://esw.w3.org/topic/SweolG/TaskForces/ CommunityProjects/LinkingOpenData

51. World Wide Web Consortium (W3C). http://www.w3.org/

52. Weiss, C., Karras, P., Bernstein, A.: Hexastore: sextuple indexing for semantic web data management. In: Proceedings of VLDB (2008)

53. Wilkinson, K.: Jena property table implementation. In: SSWS (2006)

54. Wilkinson, K., Sayers, C., Kuno, H., Reynolds, D.: Efficient RDF storage and retrieval in Jena2. In: SWDB, pp. 131-150 (2003) 